\title{
Article
}

\section{Digital Twin Geometry for Fibrous Air Filtration Media}

\author{
Ivan P. Beckman ${ }^{1, *}\left(\mathbb{D}\right.$, Gentry Berry ${ }^{2}\left(\mathbb{D}\right.$, Heejin Cho $^{2}\left(\mathbb{D}\right.$ and Guillermo Riveros ${ }^{1}$ \\ 1 Information Technology Laboratory, U.S. Army Engineer Research and Development Center, \\ Vicksburg, MS 39180, USA; guillermo.a.riveros@usace.army.mil \\ 2 Institute for Clean Energy Technology, Mississippi State University, 205 Research Blvd., \\ Starkville, MS 39759, USA; gnb26@msstate.edu (G.B.); cho@me.msstate.edu (H.C.) \\ * Correspondence: ivan.p.beckman.civ@mail.mil or ivan.p.beckman@usace.army.mil
}

Citation: Beckman, I.P.; Berry, G.; Cho, H.; Riveros, G. Digital Twin Geometry for Fibrous Air Filtration Media. Fibers 2021, 9, 84. https:// doi.org/10.3390/fib9120084

Academic Editor: Constantin Chalioris

Received: 11 November 2021 Accepted: 6 December 2021 Published: 16 December 2021

Publisher's Note: MDPI stays neutral with regard to jurisdictional claims in published maps and institutional affiliations.

Copyright: (c) 2021 by the authors. Licensee MDPI, Basel, Switzerland. This article is an open access article distributed under the terms and conditions of the Creative Commons Attribution (CC BY) license (https:/ / creativecommons.org/licenses/by/ $4.0 /)$.

\begin{abstract}
Computational modeling of air filtration is possible by replicating nonwoven nanofibrous meltblown or electrospun filter media with digital representative geometry. This article presents a methodology to create and modify randomly generated fiber geometry intended as a digital twin replica of fibrous filtration media. Digital twin replicas of meltblown and electrospun filter media are created using Python scripting and Ansys SpaceClaim. The effect of fiber stiffness, represented by a fiber relaxation slope, is analyzed in relation to resulting filter solid volume fraction and thickness. Contemporary air filtration media may also be effectively modeled analytically and tested experimentally in order to yield valuable information on critical characteristics, such as overall resistance to airflow and particle capture efficiency. An application of the Single Fiber Efficiency model is incorporated in this work to illustrate the estimation of performance for the generated media with an analytical model. The resulting digital twin fibrous geometry compares well with SEM imagery of fibrous filter materials. This article concludes by suggesting adaptation of the methodology to replicate digital twins of other nonwoven fiber mesh applications for computational modeling, such as fiber reinforced additive manufacturing and composite materials.
\end{abstract}

Keywords: single fiber efficiency; analytical filtration model; digital twin geometry; fiber geometry modeling; nonwoven nanofibrous filter media; solid volume fraction

\section{Introduction}

Our health and livelihood depend heavily upon clean breathable air, as illustrated most recently by the COVID-19 pandemic. The effort to combat the airborne spread of COVID-19 through technologies such as personal protective filter masks and HVAC filters emphasizes the importance of continued research in air filtration media [1]. The U.S. Department of Energy (DOE) has long known the importance of filter media research [2,3]. The DOE installs High Efficiency Particulate Air (HEPA) filters as the final line of defense in the containment of hazardous airborne particles of High Level Waste (HLW) storage tanks [4-7]. Continuous improvement of filter materials regarding characteristics such as the resistance to airflow and particle capture efficiency is important for personal protection from COVID-19 as well as high capacity HEPA filters. For example, a protective mask that has a low resistance to airflow and a high particle capture efficiency would function better, as more air would flow through the mask in the event of an imperfect seal, or breathing could be more comfortable for the individual, while also providing the highest possibility for capturing particles transporting COVID-19. Furthermore, as our understanding of the mechanics of filtration and particle capture increases, it becomes possible to engineer filtration mediums for specific purposes. This tailored approach would have the benefit of providing high performing filtration media in one instance, which may perform poorly in another instead of a generalized medium that performs only adequately in all situations.

Contemporary HEPA filters are manufactured with meltblown polypropylene or glass fibers into a mesh of non-woven, randomly aligned, small-diameter fibers [8]. Overall 
filtration efficiency and flow resistance are key aspects of a filter. Successful analytical models correlate filtration efficiency $\left(E_{\mathrm{F}}\right)$ and air flow resistance $(\Delta P)$ to filter thickness $(t)$, solidity $\left(\alpha_{\mathrm{f}}\right)$, fiber radius and diameter $\left(r_{\mathrm{f}}\right.$ and $\left.d_{\mathrm{f}}\right)$, volumetric air flow rate $(Q)$, filter face area $(A)$, and air viscosity $(\eta)$. It is understood that the diameter of fibers not only impacts the collection efficiency of airborne particles, but also the resistance to airflow of the filtration media as well. These concepts are illustrated in Equations (1)-(3) below and further explored in the Single Fiber Efficiency (SFE) model presented in the Appendix A $[9,10]$. Equation (1) is the end result of the SFE model. It should be noted that for the same number and length of fibers within a given volume, an increasing fiber diameter will have the effect of increasing the solidity of the media. However, the fiber diameter and solidity are not expressly dependent on each other in terms of filtration modelling, and thus are typically considered as independent characteristics and variables of the filtration medium. Thus, it may be observed from Equation (2) that flow resistance will increase with increasing solidity and decrease with increasing fiber diameter. Furthermore, a decrease in the fiber diameter results in an increase in overall collection efficiency.

$$
\begin{gathered}
E_{\mathrm{F}}=1-\exp \left(-E_{\Sigma} \alpha_{\mathrm{f}} \frac{4}{\pi d_{\mathrm{f}}} t\right) \\
\frac{4 \Delta P A r_{\mathrm{f}}^{2}}{\eta Q t}=64 \alpha_{\mathrm{f}}^{1.5}\left(1+56 \alpha_{\mathrm{f}}^{3}\right) \\
\mathrm{FOM}=\frac{-\ln \left(1-E_{\mathrm{F}}\right)}{\Delta P}
\end{gathered}
$$

The ability of a filter to maximize filtration efficiency while minimizing flow resistance is described by the filter "quality," measured by a parameter known as filter Figure of Merit (FOM). The FOM is a ratio of filtration efficiency per unit thickness divided by pressure drop per unit thickness, and is calculated as the negative of the natural log of penetration divided by the change in pressure across a filter, given in Equation (3) [10]. A higher FOM value represents a higher ratio of filtration efficiency to flow resistance. The reader is referred to the work of Brown and Hinds for a more in-depth discussion of the SFE model and a thorough overview of the mechanics and dynamics of air filtration $[10,11]$. Analytical and empirical models of air filtration media are important tools in predicting filtration efficiency, flow resistance, and filter service life [12-15]. However analytical models are not a perfect representation of reality and often struggle in their ability to describe the stochastic nature of filtration [16]. Analytical models use structured geometry to represent fibrous media, such as an ordered array of fibers oriented parallel to each other and perpendicular to the airflow. An example of this is the Kuwabara cell model [17], described in the Appendix A. However, the creation of air filter media through a melt blowing or electrospinning process results in random deviations from an ideal, homogenous structure which yields non-uniform and tortuous airflow channels. Analytical models typically use ordered fiber geometry as a foundation, and then often account for the inhomogeneity of the fibrous materials through empirical correlation factors and variables such as an effective fiber diameter, inhomogeneity factor, or effective fiber length [18,19]. It is important to note the differences that accounting for the inhomogeneity in the filtration media has in regard to the performance of the filtration media. Figure 1 illustrates this concept by plotting the pressure drops of generic media modelled using the Kuwabara and Happel cell models, Davies' empirical model, and the model presented by Spielman and Goren $[9,17,20,21]$. It is noteworthy that the Kuwabara and Happel models use ordered arrays of cylinders that are perpendicular to the flow, while the Spielman-Goren model presents four cases for fibers aligned in different orientations to the flow, allowing for different fiber geometries. Figure 1 illustrates an in-plane random fiber orientation and completely random fiber orientation with respect to the airflow, representing case 1 and case 4 for the Spielman-Goren model, respectively. However, it still cannot be asserted that the Spielman-Goren cases utilize fiber geometry that is entirely consistent with realistic media, although it may be asserted that 
the randomness incorporated into it is reminiscent. Davies' empirical model is not bound by the selection of fiber geometry due to the nature of the experiments and selection of different and common filtration mediums over a range of solidities. However, empirical models suffer from the restrictive nature of using correlations and may only be applied to situations under the exact same circumstances as their corresponding experiments.

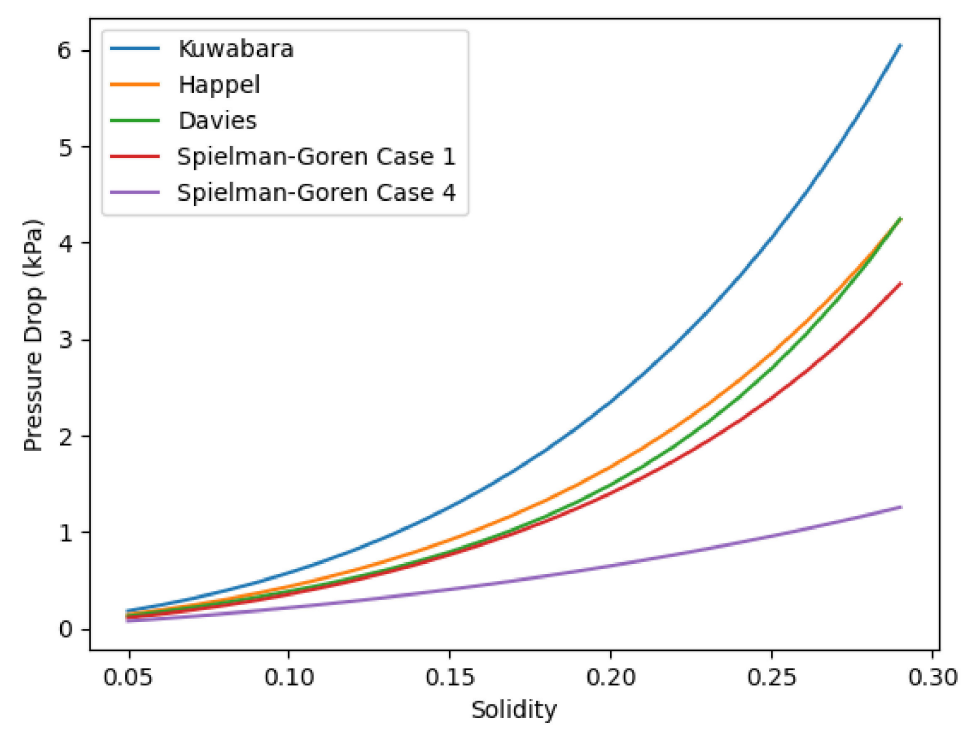

Figure 1. Pressure drop versus solid fraction for different analytical and empirical models.

Given the aforementioned drawbacks regarding the analytical and empirical models, an argument may be presented that computational modeling of air filtration media is important to complement experimental and analytical efforts. The clear benefit of a computational model's ability to account for the inhomogeneous nature of the filtration media is implicitly illustrated in Figure 1, whereby the effects of the fibrous geometry is clear on the predicted pressure drop. Thus, computational modelling presents an ideal toolset to address the difficult nature of describing the performance of fibrous filtration media and the dynamic nature of the filtration process. An example of this is that the stochastic nature of filtration may be easily incorporated into computational simulations, as well as other difficult concepts such as particle shadowing, where they may be accounted for automatically [16]. However, a drawback that should be considered is that the results from a computational simulation are not in analytical form and do not benefit from those inherent advantages. Regardless, advances in high performance computing have made Computational Fluid Dynamics (CFD) a viable tool for air filtration research. Integral to CFD modeling is the generation of digital "twin" geometry that closely replicates actual filtration media. Simply stated, without a realistic model of the fibrous geometry, the results from a CFD simulation should be questioned regarding their validity and accuracy. The purpose of this paper is to present a methodology for constructing digital twin geometry for nonwoven fibrous air filtration media, intended for use with computational modeling tools such as CFD and Finite Element Method (FEM) software.

\section{Literature Review: Virtual Three-Dimensional Geometric Models}

Efforts to build realistic digital twin geometry has progressed significantly over the past two decades. A summary list is provided in Table 1 for convenience. In 2005, Faessel et al. presented a method of generating a three-dimensional model of curved fibers to represent the random layout of cellulosic fibrous networks in low density wood-based fiberboards [22]. The authors generated director lines and curvature points as objects in Visual ToolKit (VTK) software and extruded a radius along the curve to form fibers, where the objects were converted into mesh for FEM analysis. Wang et al. in 2006 and 2007 
developed a three-dimensional virtual model for depositing straight fibers horizontally onto one another without allowing penetration [23,24]. Maze et al. in 2007 developed a three-dimensional model of compressed fiberwebs with bending fibers that prevented interfiber penetrations throughout the media, using square cross-sections to represent spun bonded media [25]. Subsequent work by the same authors enabled bending by splitting the straight fibers at intersections and angling downward on both sides of the intersection [26]. Hosseini and Tafreshi developed a C++ computer program in 2009 to generate and stack fiber mesh layers to form a three dimensional filter media model [27]. Their model allowed interpenetration of fibers with an assertion that the flow resistance and filtration efficiency are not affected by the interpenetrations as long as the exact porosity is accurately calculated. Fotovati et al. in 2009 developed a method using a FORTRAN code to construct a three dimensional model of fibers with specific in-plane orientation [28]. Fotovati's paper arranged straight unbroken fibers of identical diameter with in-plane alignments of $15^{\circ}$, $30^{\circ}$, and $45^{\circ}$ with through-plane alignment of $0^{\circ}$, for the purpose of studying the effect of fiber alignment on filtration efficiency. In 2013, Saleh et al. developed a method of producing three-dimensional geometry of disordered fibrous structures to study the effects of dendrite formation within nonwoven air filtration media [13]. Saleh's model generated random two-dimensional in-plane fiber orientations and subsequently stacked the planes to form the three-dimensional geometry similar to the previously cited work of Hosseini and Tafreshi. In 2016, Karakoc et al. modeled composite structure fiber networks as planar projections and intersections of rectangular cross-section shaped fibers [29]. Finally, in 2019 Yousefi and Tafreshi made use of Python scripting and $\mathrm{C}++$ programming to replicate electrospun fiber materials using Kelvin-Voight method of representing fibers as a series of springs and dampers $[30,31]$. The current effort presented in this work is intended to complement these accomplishments by offering a simple methodology to produce digital fibrous geometry.

Table 1. Significant efforts constructing three-dimensional digital twin air filter geometry.

\begin{tabular}{ccc}
\hline Author & Year & Description \\
Faessel et al [22] & 2005 & 3-D model generated with Aphelion software \\
and Visual ToolKit
\end{tabular}




\section{Materials and Methods: Scripting a Three-Dimensional Digital Twin}

Computational modeling of filter media begins with geometry. SpaceClaim, a fundamental module of Ansys Workbench, was used to generate the solid model. Cylindrical fibers can be created in SpaceClaim by specifying coordinates for the fiber start point, end point, and a point on the fiber surface perpendicular to the end point. While the graphical user interface is convenient to visualize geometry, SpaceClaim also has the ability to utilize a scripting feature capable of importing a text file list of coordinates for the construction of fibers. As illustrated by Figure 2, the data describing a mat of randomized fibers can be generated with a simple Python script and subsequently output as text file for use in SpaceClaim.

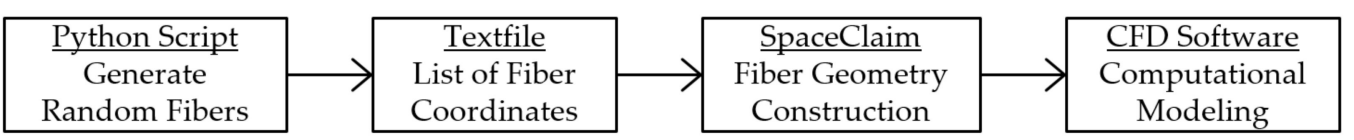

Figure 2. Methodology for random fiber generation and filter geometry construction.

A simple method for constructing three-dimensional filter geometry starts with a two-dimensional model to represent a square cut from the filter paper. Fiber endpoints are designated within the square with $\mathrm{x}$ and $\mathrm{y}$ coordinates. To achieve a random orientation for fibers originating and terminating at the edge of the square, a random number is drawn between 0 and 4 and traced clockwise around the perimeter of the square as shown in Figure $3 \mathrm{~b}$. The fiber starting point is designated at the $x$ and $y$ coordinates at that particular point on the perimeter. The fiber end point is designated in a similar method however to avoid fibers that start and end on the same edge of the square, a random number is drawn between 0 and 3 and added to the next vertex clockwise along the perimeter. The two-dimensional square is developed into three-dimensional media by randomizing the $z$ coordinates of the fiber starting and ending points within a designated media thickness. The diameter of each individual fiber is a random number driven by a user-defined distribution, such as a normal or log-normal distribution. Individual fiber volumes are calculated by the length and diameter of the fiber within the defined volume representing the total volume of the geometry model. Comparing the fiber volume to the total volume formed by the square and designated model thickness provides fiber solidity. This process is looped to add fibers until the desired solidity is achieved.

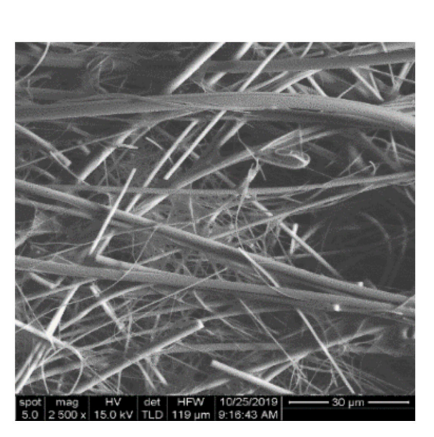

(a)

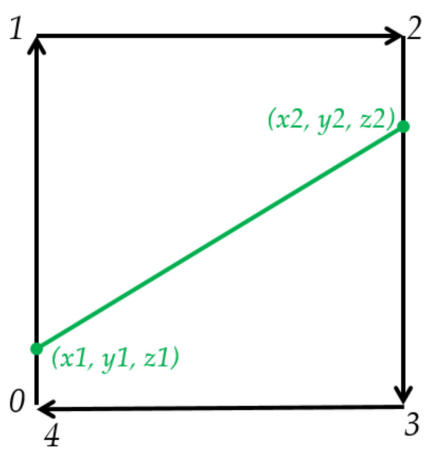

(b)

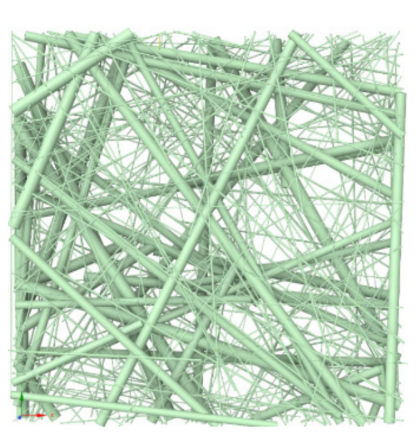

(c)

Figure 3. Fiber nonwoven mesh generation. (a) SEM image of meltblown glass fiber HEPA filter. (b) Random selection of fiber endpoints along perimeter. (c) Resulting digital twin replica.

Figure 3a shows a Scanning Electron Microscope (SEM) image of a generic meltblown glass HEPA filter media sample. Re-stating the purpose of this work more directly, with regard to the given SEM image, the goal for generating a realistic fiber geometry model of filter media would be to replicate this SEM image as closely as possible with a digital twin. From an inspection of Figure 3a, it may be seen that the glass fibers have broken ends and a bimodal distribution of fiber diameter sizes, consisting of a few very large fibers 
and many smaller fibers. By adjusting the input parameters of the Python script, digital geometry can be generated that closely resembles the SEM image. The digital geometry in Figure $3 \mathrm{c}$ was produced with similar dimensions as the SEM image, with side length $100 \mu \mathrm{m}$, thickness of $20 \mu \mathrm{m}, 50 \%$ of the fibers are terminating inside the volume, $75 \%$ of the fibers have diameters between 200 and $600 \mathrm{~nm}$ while $25 \%$ of the fibers have diameters between 2.0 and $3.5 \mu \mathrm{m}$.

One noted flaw of the result shown in Figure $3 c$ is that the fibers are allowed to interpenetrate since there is no control of the fiber orientation in comparison with previously inserted fibers inside the volume. This creates complexities for meshing software and may not result in a realistic portrayal of real filter material. A solution to this problem is to account for previous fibers already placed within the volume by evaluating intersection points with a newly generated fiber and breaking the new fiber into segments. These new fiber segments are adjusted to new coordinates in order to lay on top of previous fiber geometry. This method is depicted in Figure 4 below. A new random fiber is designated with $x$ and $y$ coordinates for the endpoints as shown in Figure 4a. The fiber can be thought of as dropping into the page. The algorithm evaluates the list of previous fiber segments and determines coordinates of potential intersecting points as shown in Figure $4 \mathrm{~b}$. In the illustrated example there are eight potential intersecting points represented by letters $a$ through $h$. Now rotating the box to view the same new fiber dropping into the box from top to bottom of the page (by rotating the $\mathrm{z}$ axis in Figure $4 \mathrm{c}$ to where the $\mathrm{y}$ axis was in Figure $4 \mathrm{~b}$ ), potential intersecting points are evaluated based upon their height and distance along the fiber. A maximum fiber slope, designated as the relaxation slope, is specified to enable fiber flexibility to conform to the necessary shape indicative of realistic materials. This is illustrated below in Figure 4c,d. Starting from the highest potential intersecting point (point $c$ in the example shown), the maximum relaxation slope is traced in both directions along the fiber. Potential intersecting points occurring below the maximum slope line are eliminated. In this illustration, points $a, f, g$, and $h$ are retained while points $b, d$, and $e$ are eliminated. The algorithm then evaluates the next highest potential intersecting point (point $g$ in this illustration) and repeats the process. As depicted in Figure 4d, point $f$ which was earlier retained is now eliminated as it falls below the maximum slope line from point $g$. The algorithm continues for all intersecting points and results in a final list (points $a, c, g$, and $h$ in this illustration). The finalized lines form the profile of the new fiber broken into segments. Segment break points are calculated at the intersections as shown in Figure 4e. In this illustration, the fiber is broken into eight segments with segment endpoints specified at the points where the segments touch.

The height ( $z$ coordinate) is determined for each segment end point by adding the radii of the new fiber segment and the previous fiber segment responsible for the spatial adjustment. Additional spacing between fibers can be added by increasing the $z$ coordinates for the new fiber segment end points. Figure 5 illustrates the process of closing the newly segmented fiber sections with a spherical joint between each new segment, resulting in a continuous, solid fiber.

By eliminating fiber interpenetrations, the digital twin nonwoven fibrous geometry is much easier to mesh since the meshing software is able to take advantage of straight fiber segments with cylindrical joints as shown in Figure 6 below. 


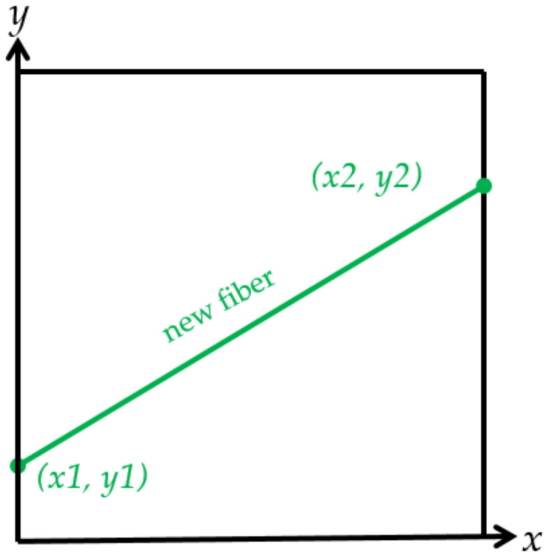

(a)

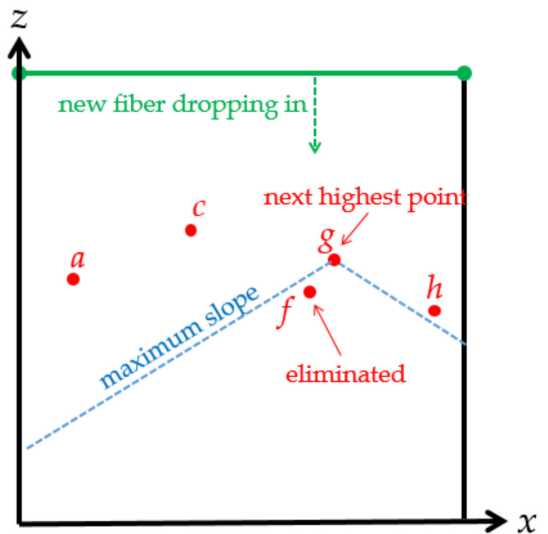

(d)

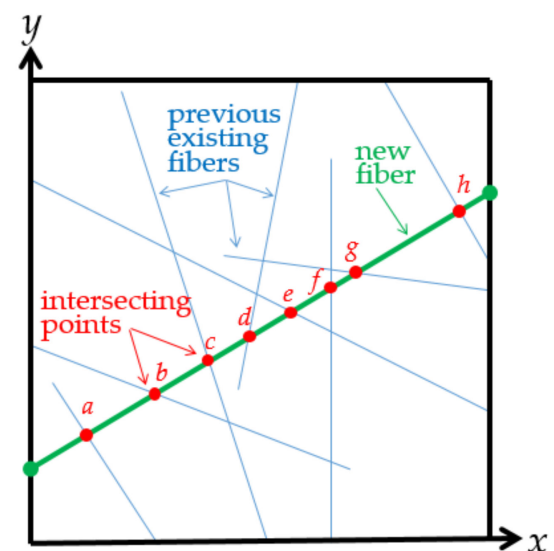

(b)

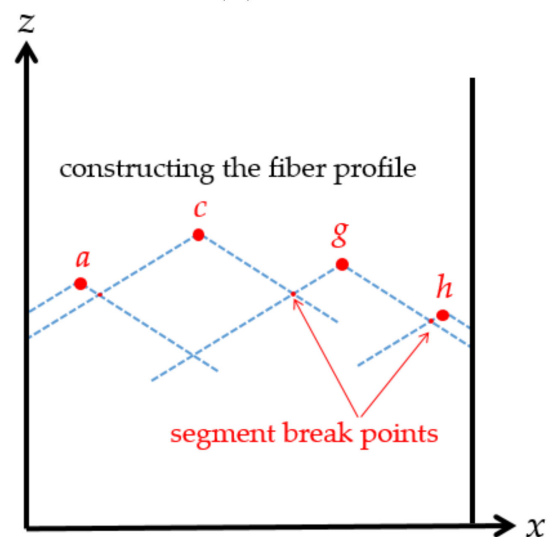

(e)

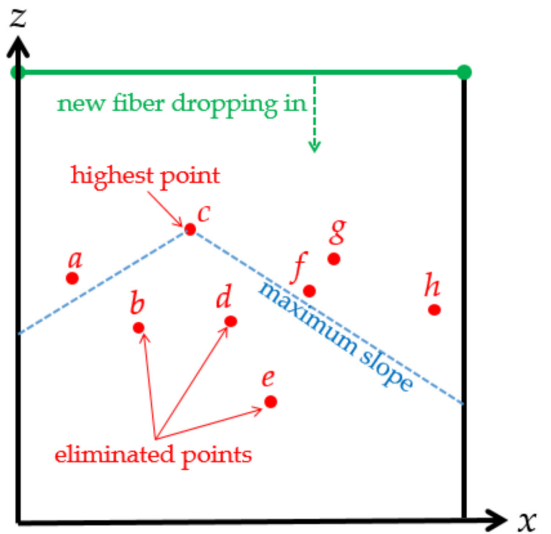

(c)

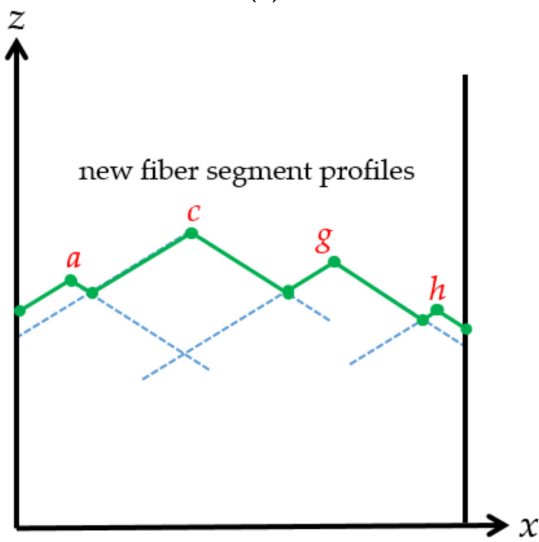

(f)

Figure 4. Process for breaking a new fiber into segments to conform to previous geometry. (a) New random fiber specified starting and ending points. (b) Potential intersection points are evaluated below the fiber. (c) Height of potential intersecting points are evaluated against maximum slope. (d) Potential intersecting points eliminated that fall below the maximum slope line. (e) Fiber segment profile is constructed from the remaining maximum slope lines. (f) New fiber segment starting and ending points are finalized.

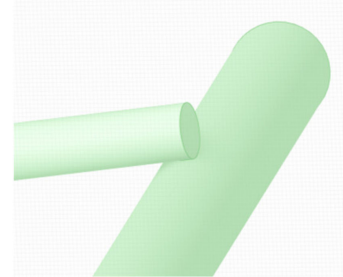

(a)

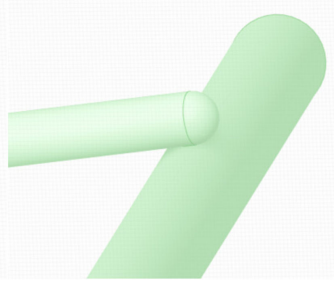

(b)

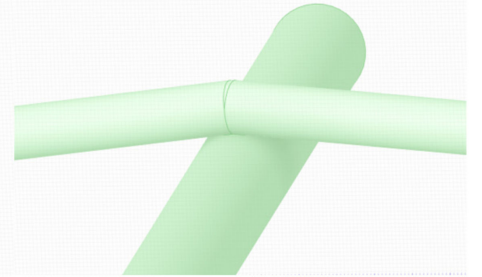

(c)

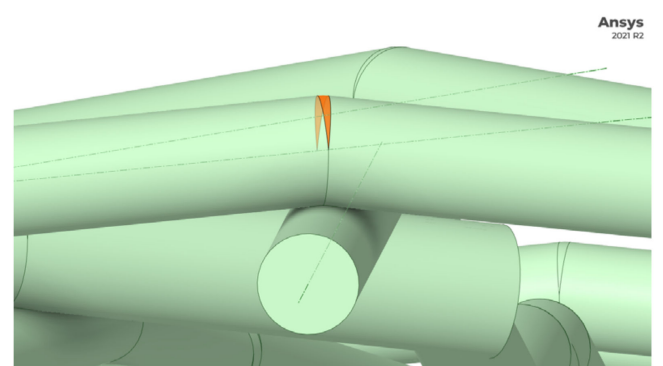

(d)

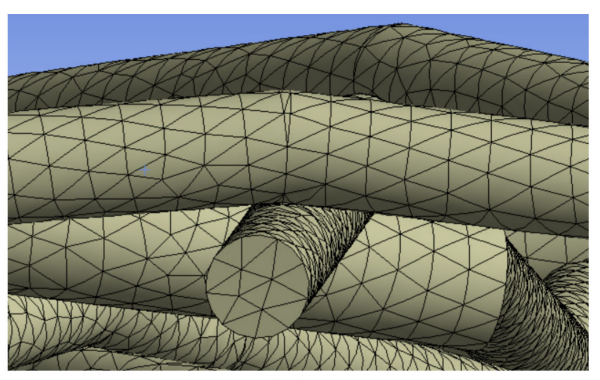

(e)

Figure 5. Spherical joints at the fiber segment connections. (a) New fiber segment resting on previous fiber. (b) Spherical joint at segment endpoint. (c) Continuation from joint. (d) Completed fiber with highlighted joint. (e) Completion of meshing. 


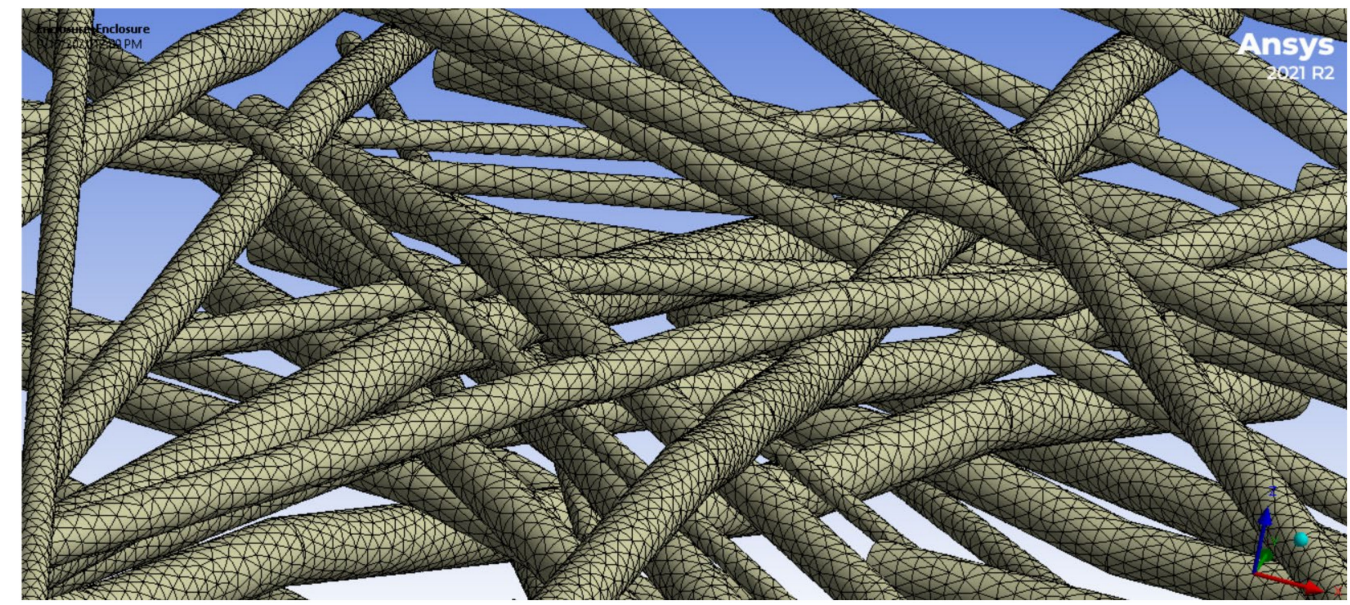

Figure 6. Non-intersecting fibers meshed with Ansys Mechanical.

\section{Results: Filter Solidity, Face Coverage, Thickness, and Flow Resistance}

The solidity, thickness, and face coverage are parameters that indicate a quantity of fibers in a filter medium. Thickness is a one-dimensional measurement. Face coverage is a two-dimensional measurement of the fiber profile normal to the direction of air flow, comparing the cross sectional coverage of the fibers with the cross sectional area of the box. The solidity is a three-dimensional concept, defined as the ratio of the total volume occupied by fibers to the total volume of the media. Figure 7 shows an example of nonwoven fibrous air filter media created with the digital twin geometry script, constructed into solid geometry with SpaceClaim, and meshed with Ansys Mechanical. The available input parameters used to adjust the digital twin geometry to match SEM images include face coverage, relaxation slope, distribution of fiber radius, and percentage of fibers that are broken inside the box. The resulting digital twin geometry can be meshed and then input into CFD software for analysis.

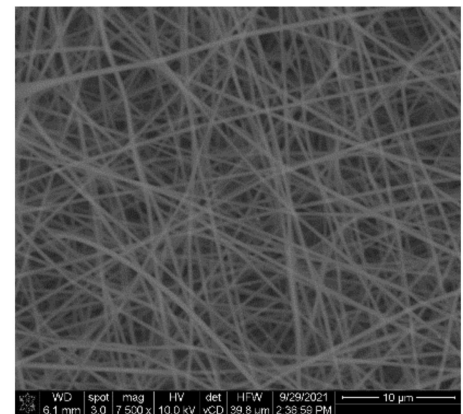

(a)

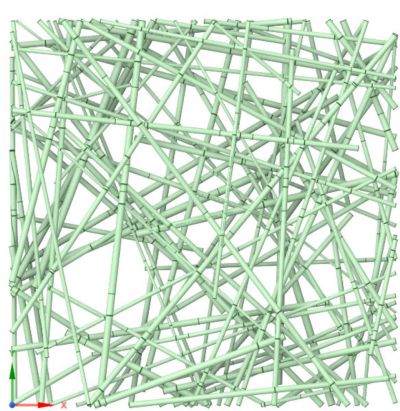

(b)

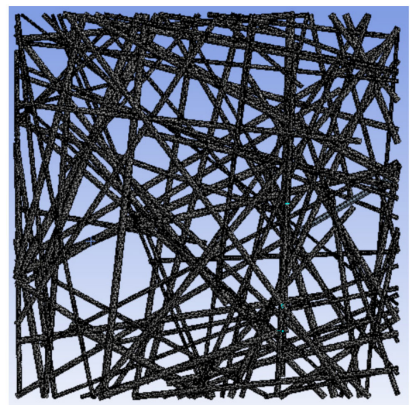

(c)

Figure 7. Non-intersecting air filter media geometry. (a) SEM image of electrospun filter media. (b) Top view as created with SpaceClaim. (c) Filter media meshed by Ansys Mechanical.

The algorithm illustrated in Figure 4 eliminates the problem of fiber interpenetrations illustrated in Figure 3. It is worth noting that the face coverage, media thickness, or flow resistance can be used as design criterion while solidity becomes a dependent parameter. The algorithm can continually add fibers on top of the filter until the desired face coverage is achieved, while filter thickness and solidity become dependent on the face coverage. Fiber flexibility is a significant factor in this method to determine the thickness and solidity, which can be adjusted with the maximum relaxation slope specified in the model. Figure 8 shows a profile view of a model built to a specified face coverage of 1.0, given various relaxation slope allowance from straight fibers with $0 \%$ slope to bent fibers with $25 \%$ slope. The solidity of these models varies from about $1.80 \%$ to $12.0 \%$ while the thickness varies 
from about $132 \mathrm{~nm}$ to $17 \mathrm{~nm}$. This illustrates the effect of relaxation slope on the overall filter thickness and solidity.

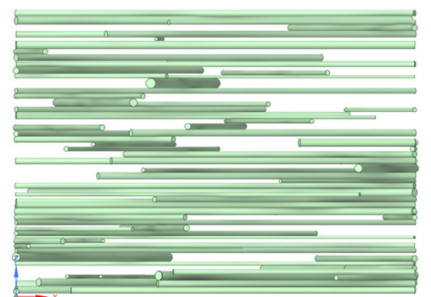

(a)

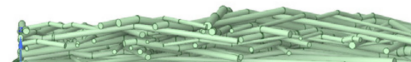

(d)

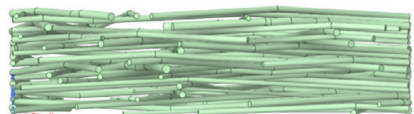

(b)

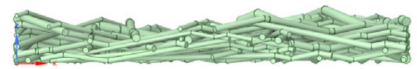

(e)

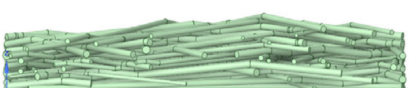

(c)

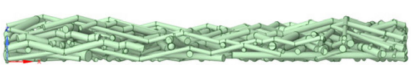

(f)

Figure 8. Profile view of fiber geometry with varied maximum slope. (a) Straight fibers (0\% slope). (b) $5 \%$ Slope. (c) $10 \%$ Slope. (d) $15 \%$ Slope. (e) $20 \%$ Slope. (f) $25 \%$ Slope.

Figure 9 shows the relationship between the specified fiber relaxation slope and resulting filter thickness and solidity for a given face coverage area. This analysis was performed on a $100 \mu \mathrm{m} \times 100 \mu \mathrm{m}$ area with mean fiber diameter of $700 \mathrm{~nm}$ and a face coverage of 2.0. It is worth noting here that since the generation of geometry is completely random, the characteristics of the generated media, such as solidity, will be unique by definition for every generated sample, even with identical inputs. This is illustrated in Figure 9 by use of the error bars, which depict a confidence level of $95 \%$ and were reduced from the output of the script. If the filter solidity, filter thickness, and fiber diameters of a specific piece of fibrous medium are known, the digital twin geometry can be iteratively tuned to match its parameters by adjusting the relaxation slope or adding fiber spacing between fibers.

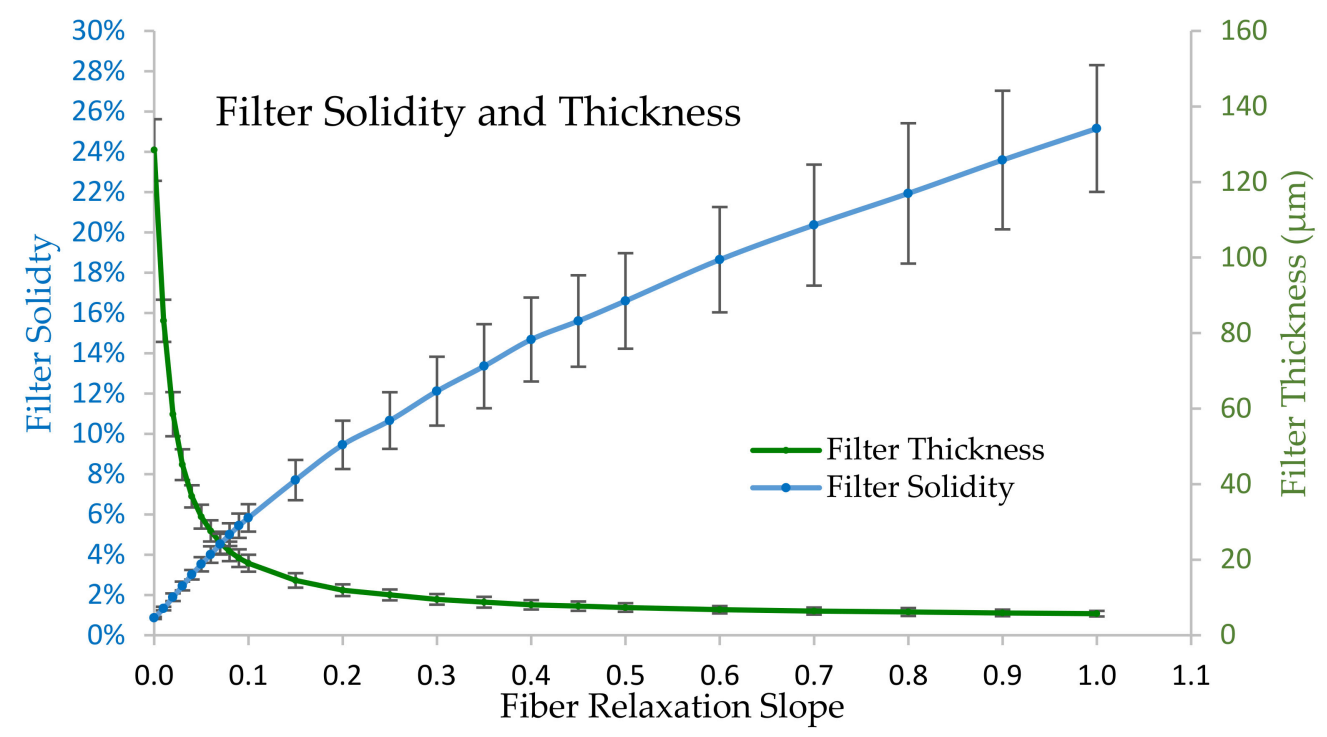

Figure 9. Filter solidity vs. maximum slope of segments. The data represents mean values of samples drawn at each fiber relaxation slope. Error bars represent a 95\% confidence level.

In addition to face coverage area, the overall filter thickness can be used as design criteria by utilizing a while loop to continually add fibers on top of the previous layers until the desired thickness is achieved. A simple method is to measure filter thickness by the height of the tallest fiber endpoint. 


\section{Discussion: Analytical Modeling of Digital Twin Filter Geometry Performance}

The SFE model is a well-known analytical tool that has been traditionally prevalent in literature which may be used to estimate the total filtration efficiency of filter media based on a calculated particle capture efficiency of a single, isolated fiber positioned perpendicular to the direction of flow $[8,27,28]$. Yousefi and Tafreshi in 2020 demonstrated the use of the SFE model to predict the filtration efficiency, flow resistance, and FOM of digital twin electrospun filter media embedded with spacer particles [30]. Using their method as a guide, the SFE model can be applied to predict the filtration efficiency, flow resistance, and FOM for our digital twin filter. The SFE model may be easily incorporated into the script to automatically generate geometry that yields desired analytical results, such as total filter efficiency and air flow resistance. Key filter material input factors include solidity, thickness, and fiber diameter. Key aerosol factors include particle diameter and particle density, while key airflow factors include air velocity, density, temperature, and viscosity. The SFE model is useful for describing the collection efficiency based on a uniform fiber diameter and for a single particle size. However, the SFE model introduces inherent error since both aerosols and fibers typically follow a statistical distribution, i.e., normal or log-normal. This limitation can be addressed by describing the situation with representative variables, such as a mean fiber diameter or mean particle diameter. Conversely it is also possible to select a representative fiber diameter and analyze the filtration media over a range of particle sizes. This is a particularly useful analysis and allows for the evaluation of the Most Penetrating Particle Size (MPPS). The MPPS is simply the particle size that the filter is least efficient at collecting under a specified set of circumstances. It is worth noting that this value is dynamic and will change with changing values such as the air flow velocity and mean fiber diameter. The flow resistance is measured as the air pressure differential between the upstream and downstream air flows relative to the filter media, which depends on the drag induced by the fibers in the media as well as any particles collected onto those fibers. The efficiency and flow resistance then determine the filter's FOM.

\subsection{Example Digital Media Generation}

Figure 10 shows an example nonwoven air filter media digital twin with dimensions of $100 \mu \mathrm{m} \times 100$ perpendicular to the direction of flow. All fibers span the box with four-sided symmetry. The relaxation slope was set to $15 \%$ with a constant fiber radius of $1.4 \mu \mathrm{m}$, for a face coverage area of 4.0 with no additional spacing between fibers. This design resulted in the production of 337 fibers with 3204 segments, a solidity of $9.48 \%$, and a thickness of $47.0 \mu \mathrm{m}$. With a specified airflow face velocity of $2.5 \mathrm{~cm} / \mathrm{s}$, the analytical model predicted flow resistance of $21.2 \mathrm{~Pa}$. The total filter efficiency graphs show a minimum efficiency of $41.9 \%$ for aerosol particles with diameter of $215.4 \mathrm{~nm}$, resulting in a FOM rating of $0.01972 \mathrm{~Pa}^{-1}$. However, for $300 \mathrm{~nm}$ diameter particles, the filter media achieves an efficiency of $45.0 \%$ with a FOM rating of $0.0212 \mathrm{~Pa}^{-1}$.

For clean filtration media, the flow resistance is dependent on the airflow parameters and independent of the aerosol characteristics, although it is worth noting that the characteristics of the aerosol will determine how the flow resistance changes as the particles are captured. Therefore, the flow resistance for clean filter media, measured as the pressure differential between the upstream and downstream airflows relative to the filter media, can be analytically predicted without yet knowing filter efficiency or aerosol characteristics. Similar to fiber face coverage and filter thickness, the flow resistance can be used as a design parameter for generation of digital filter geometry by setting a while loop to continue adding fibers on top of the filter until the specified maximum pressure drop is achieved. 


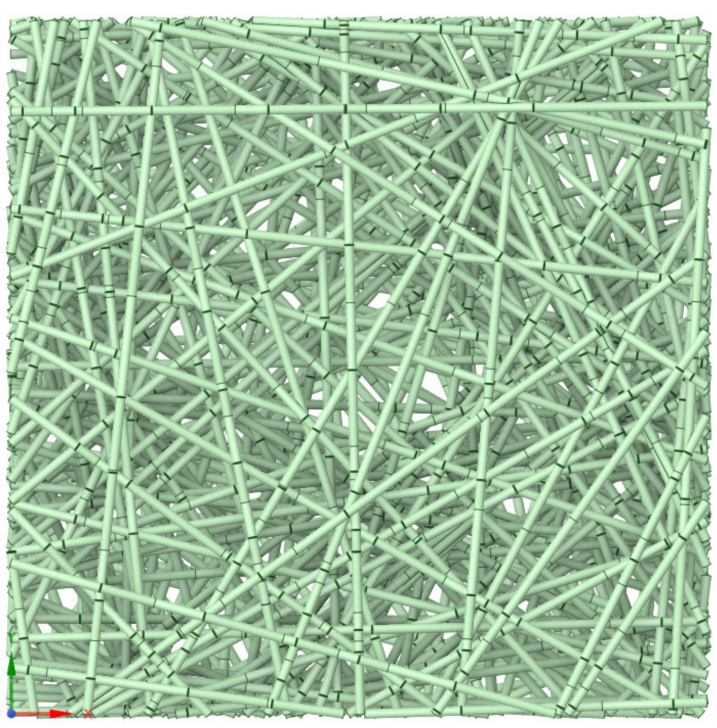

(a)

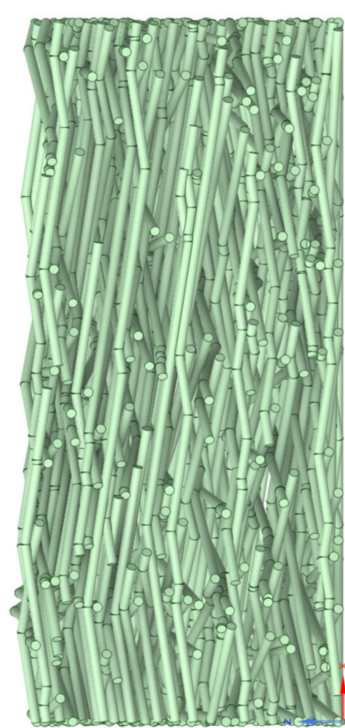

(b)

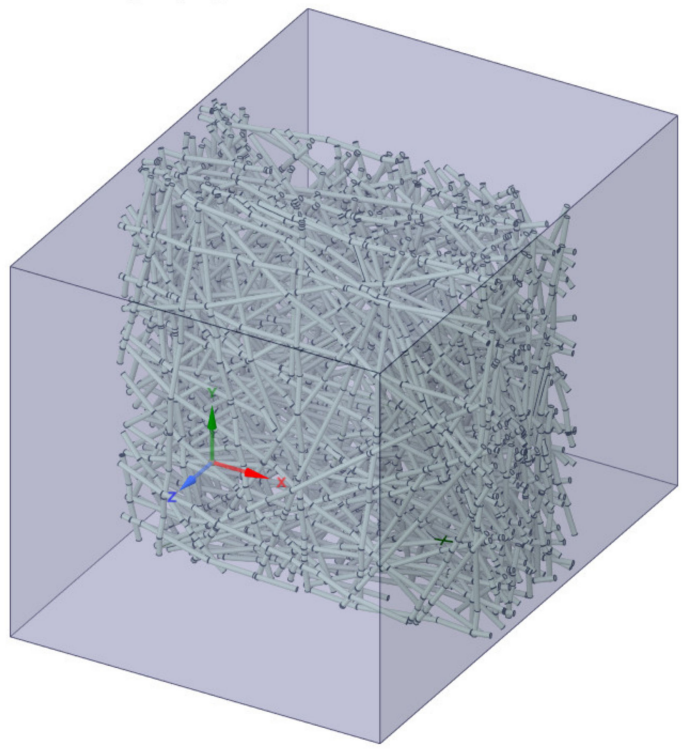

(c)

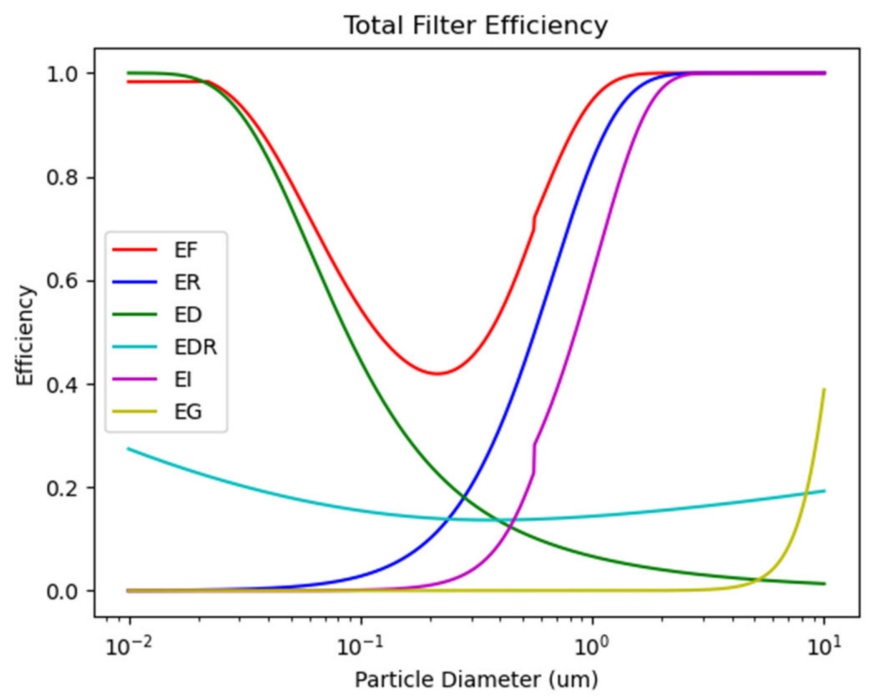

(d)

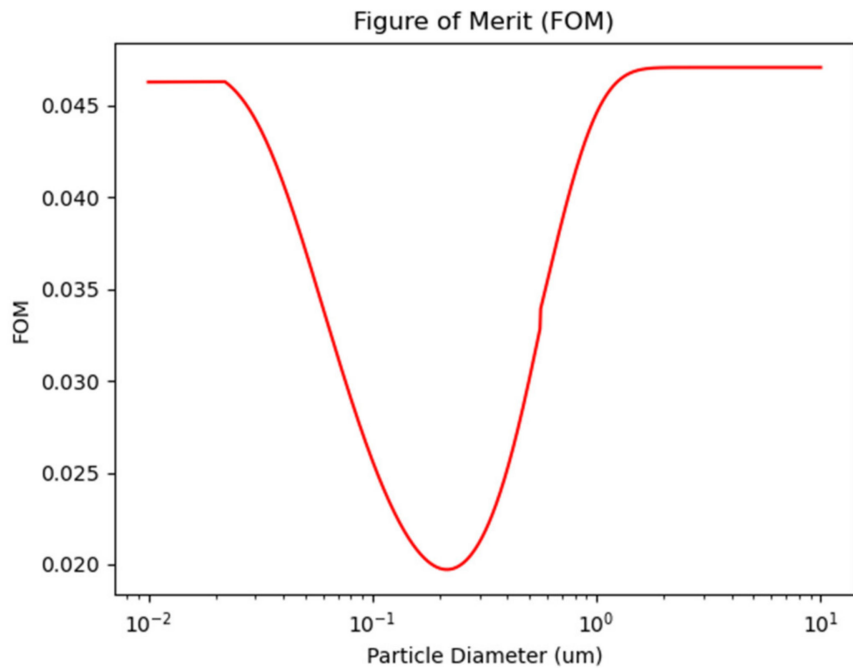

(e)

Figure 10. Analytical modeling of digital twin. (a) Top view of face area perpendicular to flow. (b) Side view showing filter thickness. (c) Geometry loaded into enclosure for CFD modeling. (d) Total filter efficiency shown by SFE model components. (e) Resulting Figure of Merit.

\subsection{Maximum HEPA Flow Resistance}

By definition, HEPA filters are required to maintain $99.97 \%$ filtration efficiency for $300 \mathrm{~nm}$ diameter particles with a maximum flow resistance of $320 \mathrm{~Pa}$ at ambient conditions with air velocity of $2.5 \mathrm{~cm} / \mathrm{s}$ [37]. For illustrative purposes, using the HEPA specified maximum flow resistance as the driving criteria, a digital twin geometry sample was created. Figure 11 shows the digital twin sample, which has square side dimensions of $20 \mu \mathrm{m}$, fiber diameters of $500 \mathrm{~nm}$, a fiber relaxation slope of $15 \%$, and an airflow velocity of $2.5 \mathrm{~cm} / \mathrm{s}$ at standard conditions. The digital twin geometry algorithm to generate an original geometry, calculate the resulting predicted flow resistance, and iterate the process until the predicted flow resistance reached $320 \mathrm{~Pa}$. The resulting filter slice had 884 fibers with 5494 segments, thickness of $68.5 \mu \mathrm{m}$, a face coverage of 19.3, and a solidity of $11.17 \%$. The minimum filtration efficiency was $99.97 \%$ for the most penetrating particle size of $153.5 \mathrm{~nm}$ with a FOM rating of $0.00312 \mathrm{~Pa}^{-1}$. Thus, according to the SFE model and Davies' pressure drop model, a piece of filtration media with the same characteristics and structure 
as the digital twin geometry would meet HEPA standards with a filtration efficiency of $99.999 \%$ occurring at particle size of $300 \mathrm{~nm}$.

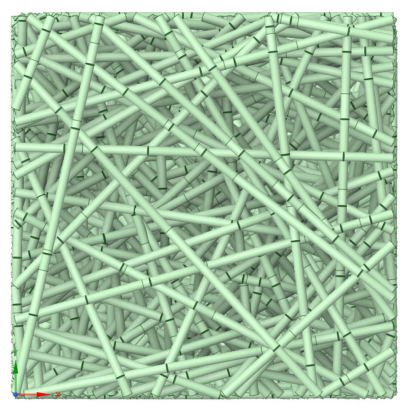

(a)

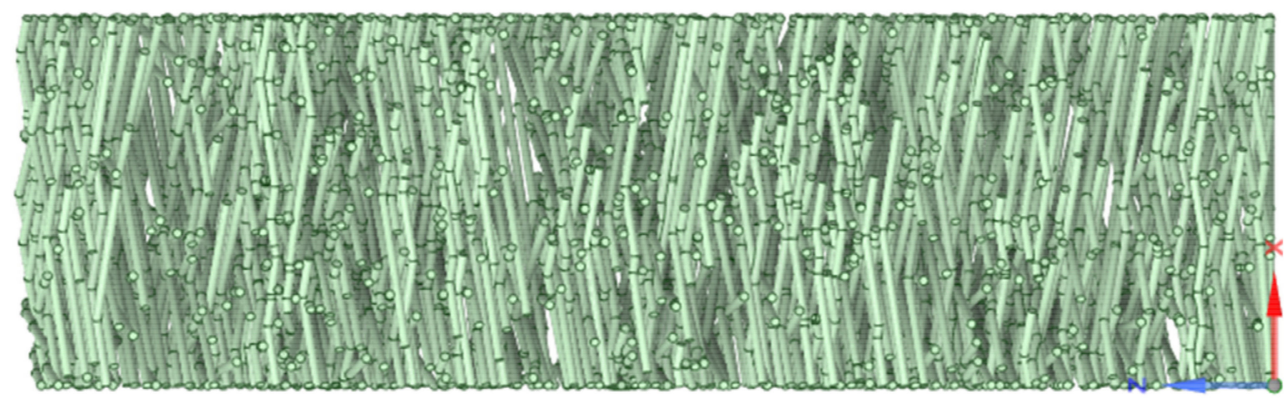

(b)

Figure 11. Digital twin HEPA filter built to 320 Pa flow resistance. (a) Top view of face area perpendicular to flow. (b) Side view showing filter thickness.

\subsection{Minimum HEPA Filtration Efficiency}

A second iteration was performed with a while loop to iterate the process until the total filter efficiency achieved $99.97 \%$ in accordance with HEPA standards. The resulting filter slice, again with fiber diameters of $500 \mathrm{~nm}$, had 575 fibers with 3791 segments, thickness of $42.3 \mu \mathrm{m}$, a face coverage of 12.76 , and a solidity of $11.98 \%$. This achieved a $99.97 \%$ efficiency at particle size of $300 \mathrm{~nm}$ with a flow resistance of $223 \mathrm{~Pa}$ and resulting FOM of $0.00448 \mathrm{~Pa}^{-1}$. Filtration media with the same characteristics and structure would meet HEPA standards. The resultant digital replica for this iteration with its corresponding total filtration efficiency graph is shown in Figure 12.

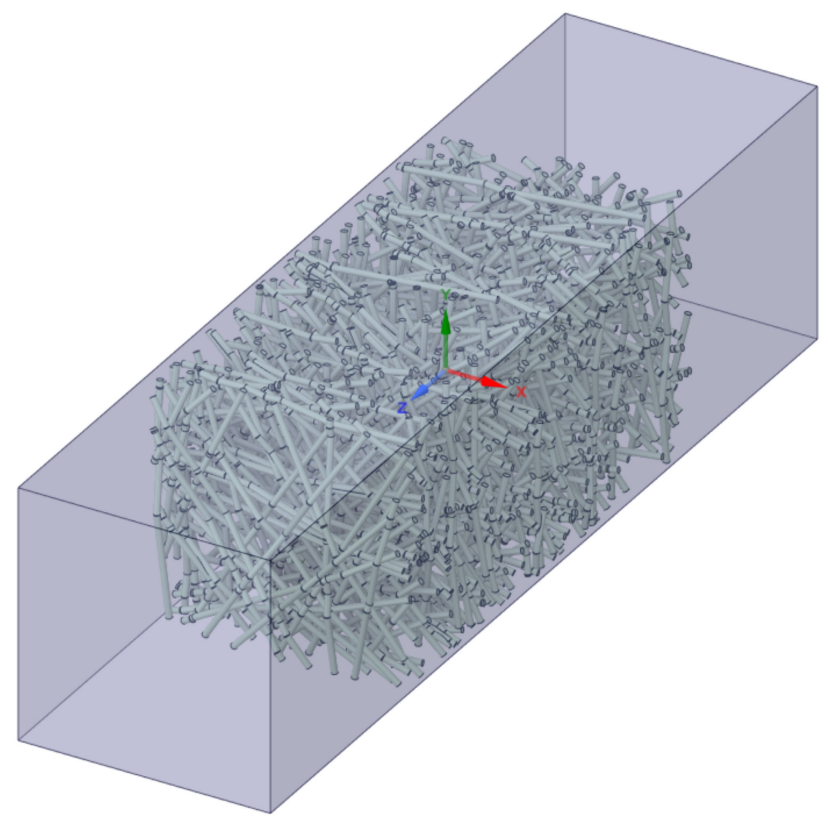

(a)

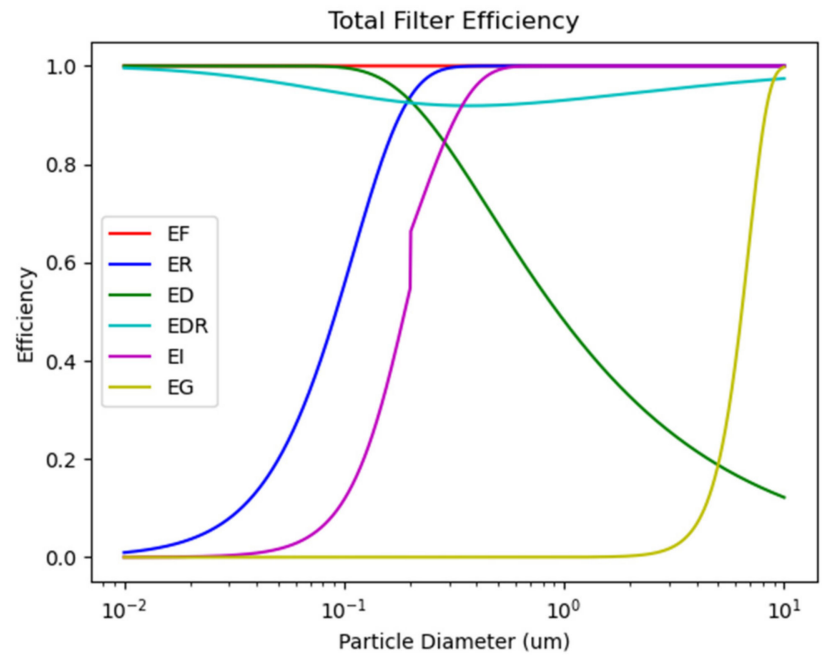

(b)

Figure 12. Digital twin HEPA filter built to $99.97 \%$ efficiency. (a) Geometry loaded into enclosure for CFD modeling. (b) Total filter efficiency shown by SFE model components.

\subsection{Fiber Diameter Sensitivity Analysis}

A sensitivity analysis was performed by varying the fiber diameter of the digital twin geometry, ranging from $400 \mathrm{~nm}$ to $1.6 \mu \mathrm{m}$ while using a relaxation slope of $15 \%$ until achieving a face coverage of 4.0. The error bars were calculated similarly to those in 
Figure 9, with a confidence level of $95 \%$. It is interesting to note that for a particle size of $300 \mathrm{~nm}$, the total filtration efficiency increased from $75.8 \%$ to $99.6 \%$ by reducing the fiber diameter from $1.6 \mu \mathrm{m}$ to $400 \mathrm{~nm}$. The solidity changed from $12.24 \%$ to $8.88 \%$ over this same range of diameter sizes. This illustrates that filtration media with different characteristics, such as fiber diameter or solidity, will perform differently regarding differently sized particles. The sensitivity analysis for total filter efficiency and solidity based on fiber diameter size is depicted in Figure 13.

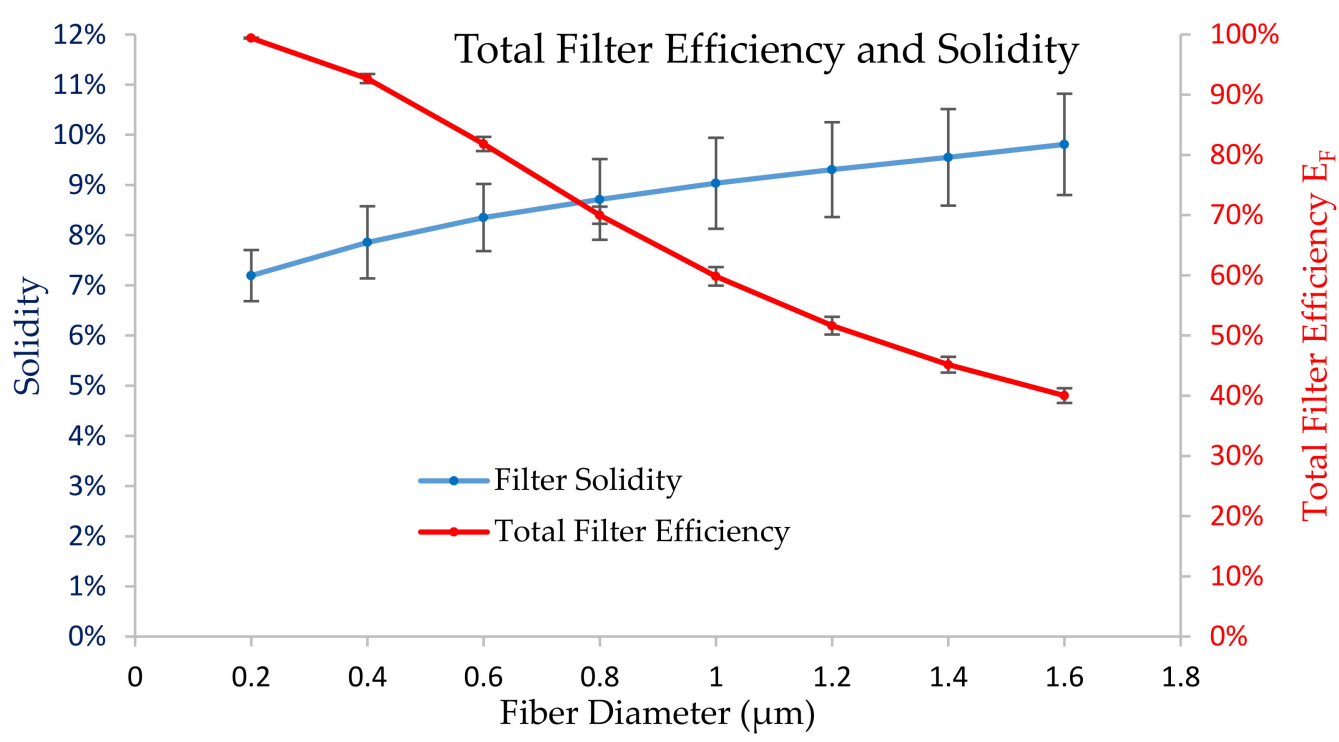

Figure 13. Sensitivity analysis for total filter efficiency and filter solidity. The data represents mean values of samples drawn at each diameter size. Error bars represent a 95\% confidence level.

\section{Conclusions}

This article presents a simple method for constructing digital twins for nonwoven fibrous air filtration media using a Python script and geometry software package. Input parameters are easily adjusted to tune the resulting digital twin geometry into realistic replicas of electrospun or meltblown nonwoven fibrous filter media. This article also demonstrates the usefulness of the SFE analytical model along with a pressure drop model to predict the filtration efficiency and flow resistance of a digital twin filter, and how it may be applied with the presented algorithm to generate a geometry to yield desired predicted characteristics. To illustrate this concept, a HEPA quality digital twin filter slice was created and analyzed. A visual comparison between an SEM image of electrospun media and a generated digital twin shows good agreement regarding a similarity in their fiber geometries. Furthermore, the ability to use the SFE model along with the script used to generate the digital twins was shown to be useful in estimating the performance of the generated geometry, or useful in generating geometry with a specific estimated performance in mind. Recommended future efforts include the refinement of this digital twin geometry creation algorithm and CFD analysis of air filtration media with the goal of matching experimental, analytical, and computational models of air filtration. This method of nonwoven fiber mesh digital twin creation may also be extended to other computational models requiring semi-random fiber placement, to include mechanical and thermal evaluation of fiber reinforced composite materials produced by additive manufacturing [38]. Given this, the primary advantage of this geometry generation method may be stated to be the simplicity and ease of adjusting parameters to achieve realistic random, semi-random, and aligned fiber orientations. 
Author Contributions: Conceptualization H.C.; Formal analysis I.P.B., G.B., H.C.; Funding acquisition G.R.; Investigation I.P.B., G.B.; Methodology H.C., G.R, I.P.B., G.B.; Project administration G.R.; Resources G.R.; Supervision H.C., G.R.; Validation G.B., H.C.; Visualization I.P.B., G.B.; Writingoriginal draft I.P.B.; Writing-review and editing I.P.B., G.B., H.C., G.R. All authors have read and agreed to the published version of the manuscript.

Funding: The authors acknowledge the financial support provided by the U.S. Army Engineer Research and Development Center under Work Unit "Innovative Hybrid Simulations for Additive Manufacturing." Permission was granted by the Director, Information Technology Laboratory to publish this information.

Data Availability Statement: The data presented in this study are available in referenced materials.

Conflicts of Interest: The authors declare no conflict of interest.

\section{Appendix A}

\section{Appendix A.1 Analytical Modeling of Air Filtration}

Classical modeling of fibrous air filter media consists of two-dimensional analytical representations of aerosol flow around fibers. The Single Fiber Efficiency model is a well-known analytical tool present in literature and relatively simple to implement that predicts the total filtration efficiency of filter media based on the particle capture efficiency of a single, isolated fiber positioned perpendicular to the direction of flow $[10,18,39]$. As illustrated by Figure A1, SFE models consider the number of particles approaching the fiber geometrically incident to the fiber's cross section. Classical SFE models estimate the percentage of approaching particles that are captured by the fiber.

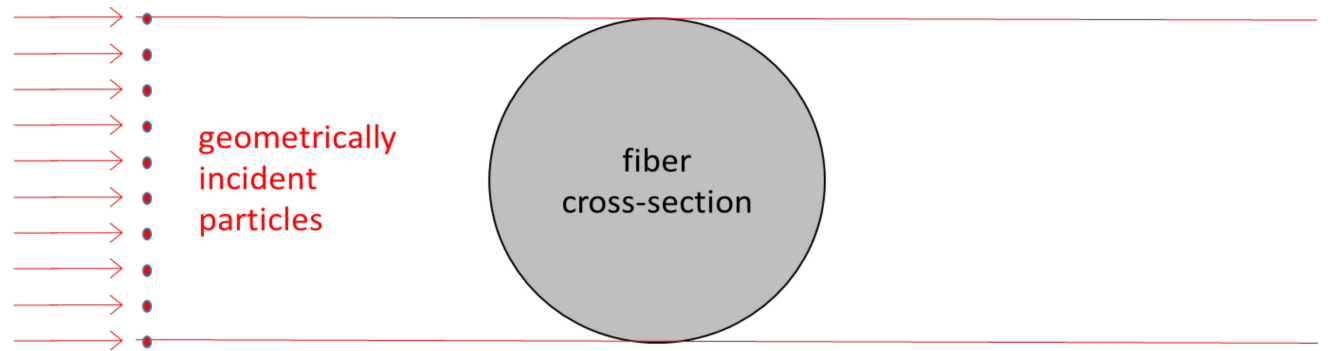

Figure A1. Geometrically incident particles approaching a fiber cross section.

\section{Appendix A.2 Kuwabara Cell Model}

Prior to 1959 the single fiber model assumed each fiber acted independently within an infinitely sized flowfield. Two authors in 1959, Kuwabara and Happel, separately improved the single fiber model to account for the effects of neighboring fibers $[17,20]$. Kuwabara developed a cell model to represent cylinders and their surrounding space as cells as shown in Figure A2.

Airstreams flow around nanometer diameter fibers differently than expected by the noslip boundary condition and cannot rely on the assumption of a continuum. As the radius of the fiber approaches the mean free path of air molecules, the no-slip boundary condition no longer accurately portrays air-fiber dynamics. The fiber surface allows a slipping condition for air molecules, which must be taken into account during computational fluid dynamics modeling attempts. The solid volume fraction $\alpha_{\mathrm{f}}$ of a filtration media is the fraction of the volume of fiber and deposited particles to the total volume of the filter media. This is also known as packing density and is the complement of the media's porosity. The dynamics of aerosol particles must be understood in order to apply a filtration model. It is worth noting that filters are primarily designed for laminar airflow which is indicated by a relatively low Reynolds number of less than 2000 [40].

The Kuwabara cell model uses the stream function as a biharmonic equation in polar coordinates. Particles are assumed to travel precisely along the flow streamlines, and any particle following a streamline that is within one particle radius to the filter fiber will 
touch the fiber and become intercepted and deposited onto the fiber. Kuwabara's model calculated filtration efficiency by relating the solid volume fraction and ratio of the radii for the particle and fiber. The model assumes the flow velocity is slow enough to neglect the inertial terms of the Navier-Stokes equation when the representative Reynolds number $(\mathrm{Re})$ is 0.05 or less. The representative Reynolds number is shown in Equation (A1).

$$
\operatorname{Re}=\frac{\rho_{\mathrm{g}} d_{\mathrm{f}} U}{\eta}
$$

The Kuwabara cell model assumes that the radial and tangential velocities vanish at the fiber surface, the vorticity at the cell boundary cancels with the vorticity of the adjacent fiber cell, and the radial velocity along the cell boundary is a function of $\cos (\theta)$ [41].

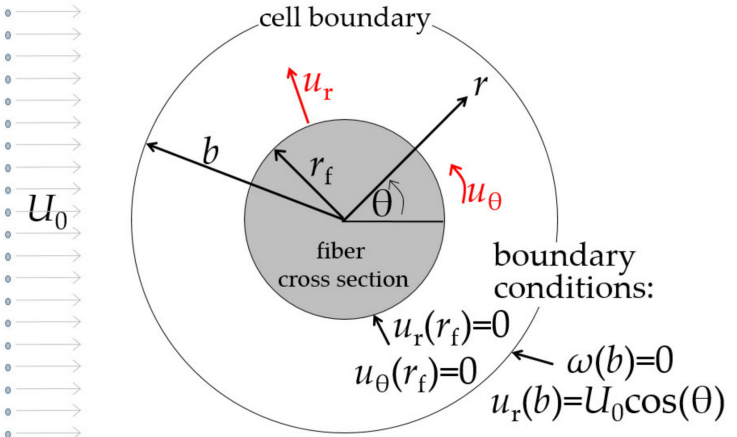

(a)

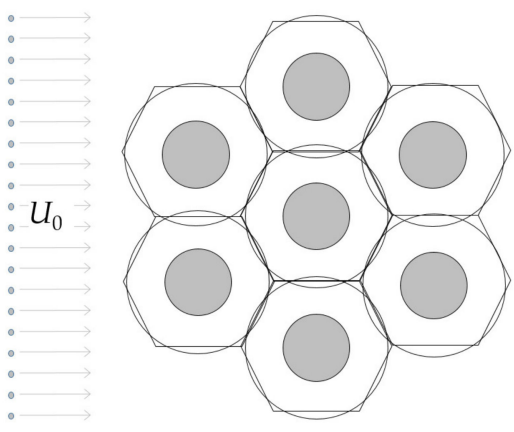

(b)

Figure A2. Kuwabara cell model for single fiber efficiency; (a) Kuwabara single fiber with boundary conditions; (b) Kuwabara flow field arrangement of fiber cross sections.

Table A1. Kuwabara cell model.

\begin{tabular}{ccc}
\hline & Symbol and Description & $\begin{array}{c}\text { Calculations and } \\
\text { Boundary Conditions }\end{array}$ \\
\hline$\psi$ & Stream function & $\nabla^{4} \psi=0$ \\
$\nabla^{2}$ & Vector Laplacian & $\nabla^{2}=\frac{\delta^{2}}{\delta r^{2}}+\frac{1}{r} \frac{\delta}{\delta \theta}+\frac{1}{r^{2}} \frac{\delta^{2}}{\delta \theta^{2}}$ \\
$\alpha_{\mathrm{f}}$ & Solid Volume Fraction & $b=\frac{r_{\mathrm{f}}}{\sqrt{\alpha_{\mathrm{f}}}}$ \\
$b$ & Distance to boundary & $v=\frac{U_{0}}{\left(1-\alpha_{\mathrm{f}}\right)}$ \\
$v$ & Mean velocity inside cell & $u_{\mathrm{r}}(b)=u_{0} \cos \theta$ \\
$u_{\mathrm{r}}$ & Radial velocity & $u_{\mathrm{r}}\left(r_{\mathrm{f}}\right)=0$ \\
$u_{\theta}$ & Tangential velocity & $u_{\theta}\left(r_{\mathrm{f}}\right)=0$ \\
$\omega$ & Vorticity & $\omega=-\nabla^{2} \psi$ \\
& & $\omega(\mathrm{b})=0$ \\
\hline
\end{tabular}

The Kuwabara hydrodynamic factor shown in Equation (A2) is a dimensionless number derived entirely from the filter solidity and is significant for the prediction of the interception component of the SFE model.

$$
\mathrm{Ku}=-\frac{1}{2} \ln \alpha_{\mathrm{f}}-\frac{3}{4}+\alpha_{\mathrm{f}}-\frac{\alpha_{\mathrm{f}}^{2}}{4}
$$

The solution to the Kuwabara model in cylindrical polar coordinates is shown as Equation (A3) [41].

$$
\psi=\frac{v r}{2 \mathrm{Ku}}\left(2 \ln \frac{r}{r_{\mathrm{f}}}-1+\alpha_{\mathrm{f}}+r_{\mathrm{f}}^{2}\left(1-\frac{\alpha_{\mathrm{f}}}{2}\right)-\frac{\alpha_{\mathrm{f}}}{2} \frac{r^{2}}{r_{\mathrm{f}}^{2}}\right) \sin \theta
$$


On the cell boundary where $r=b$ in Figure A2, the stream function has the solution shown in Equation (A4).

$$
\psi=v b \sin \theta=v y
$$

The Kuwabara cell model enables the calculation of streamflow around a single fiber, while accounting for the effects of its neighboring fibers, with geometrically incident particles that are assumed to follow the streamflow paths. This is the basic foundation of the SFE model, which analyzes particle motion in relation to flow streamlines around a fiber cross section and predicts the percentage of particles captured by the fiber compared to all particles approaching the fiber on geometrically incident streamlines as shown in Figure A3.

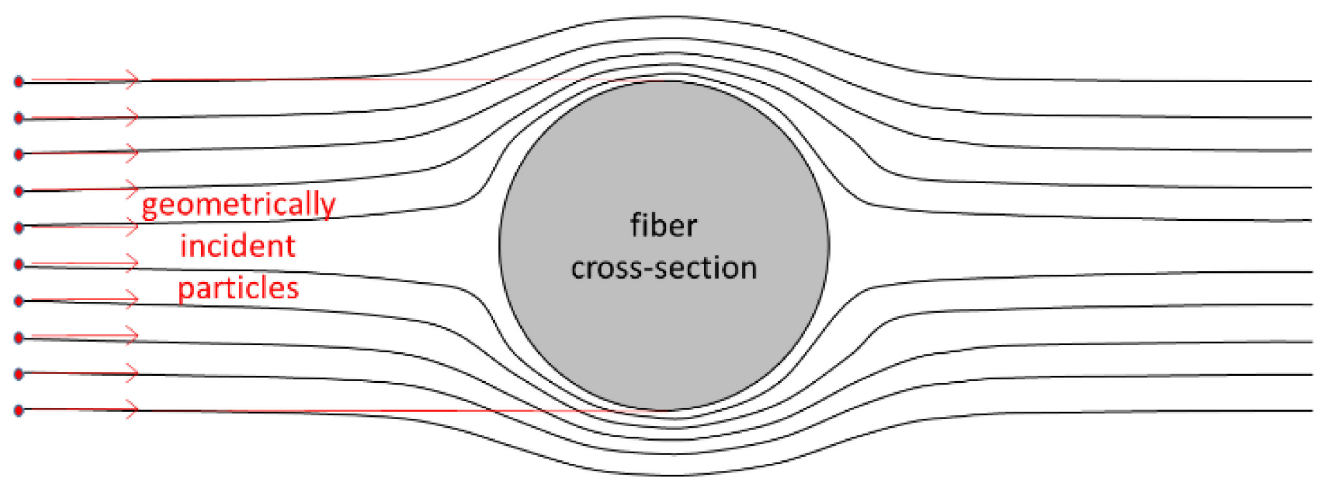

Figure A3. Single fiber flow field stream lines.

\section{Appendix A.3 Particle Deposition Mechanics}

In order to understand and apply the SFE model and other filtration models, the dynamics of aerosol particles must be understood. Lee and Liu, along with other authors, refined and expanded the SFE model to account for multiple particle deposition mechanics to include interception, inertial impaction, Brownian diffusion, gravitational settling, and electrostatic forces $[18,42,43]$. Particles that contact a fiber by these deposition mechanics are usually assumed to attach and remain affixed to the fiber through Van der Waals force. Figure A4 shows as an example three particles captured by interception, diffusion, and inertial impaction, as well as two particles that escaped capture by the fiber. The particles that escaped capture are known as penetrants.

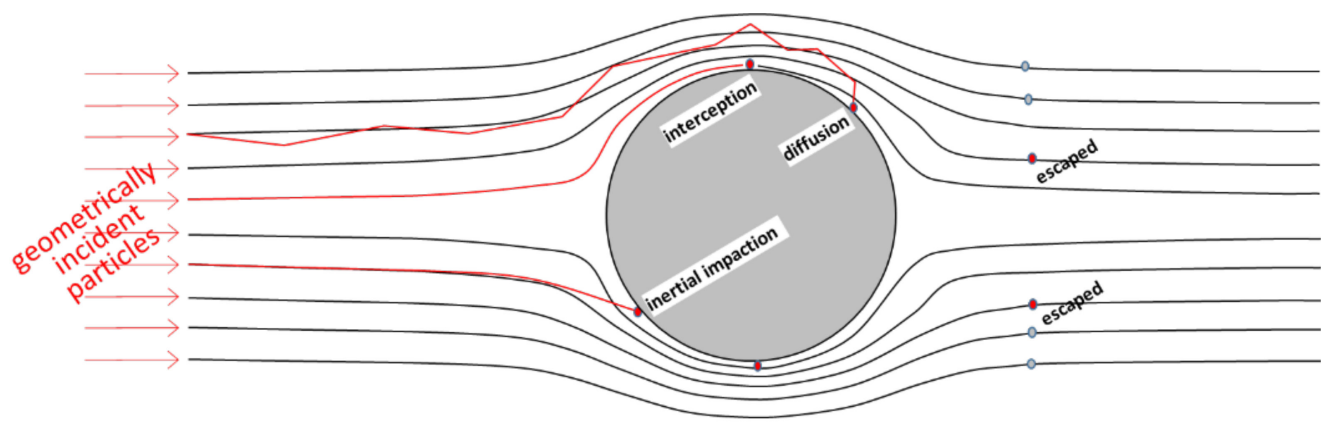

Figure A4. Particle deposition mechanics.

\section{Appendix A.4 Single Fiber Penetration and Efficiency}

Single fiber penetration, designated as the variable $P_{\Sigma}$, is the percentage of geometrically incident particles that approached the fiber incident to the fiber cross section but escaped past the fiber and avoided collection. The single fiber efficiency, $E_{\Sigma}$, is the comple- 
ment of $P_{\Sigma}$ and is defined as the percentage of incident particles approaching the fiber that become captured, given in Equation (A5).

$$
E_{\Sigma}=1-P_{\Sigma}=\left(c_{\text {in }}-c_{\mathrm{out}}\right) / c_{\mathrm{in}}
$$

As suggested by the symbol, $E_{\Sigma}$ is a combination of the component efficiencies for each deposition mechanic: Interception Efficiency $\left(E_{\mathrm{R}}\right)$, Diffusion Efficiency $\left(E_{\mathrm{D}}\right)$, Interception of Diffusing Particles Efficiency $\left(E_{\mathrm{DR}}\right)$, Inertial Impaction Efficiency $\left(E_{\mathrm{I}}\right)$, Gravitational Efficiency $\left(E_{\mathrm{G}}\right)$, and Electrostatic Efficiency $\left(E_{\mathrm{E}}\right)$. For typical air filter conditions with laminar air flow over a fiber and typical dust particle density while neglecting electrostatic effects, the size of the aerosol particle indicates the predominant capture mechanism as shown in Table A2 [10]. It is worth noting that many alternative analytical model versions for the deposition mechanics and flow resistance exist. The SFE model presented in this paper is based primarily on the SFE model presented by Hinds [10].

Table A2. Particle capture mechanisms based on particle size.

\begin{tabular}{cccl}
\hline Mechanism & $\begin{array}{c}\text { Particle } \\
\text { Size }\end{array}$ & $\begin{array}{c}\text { Particle } \\
\text { Diameter }(\mu \mathrm{m})\end{array}$ & \multicolumn{1}{c}{ Explanation } \\
\hline$E_{\mathrm{D}}$ Diffusion & Very Small & $0<d_{\mathrm{p}}<100$ & $\begin{array}{l}\text { Particles stray from flowlines by Brownian } \\
\text { diffusion and collide with fibers }\end{array}$ \\
$E_{\mathrm{R}}$ Interception & Medium & $100<d_{\mathrm{p}}<200$ & $\begin{array}{l}\text { Particles follow flowlines and collide with fibers } \\
\text { within one radius of flowline }\end{array}$ \\
$E_{\mathrm{I}}$ Inertial Impaction & Large & $d_{\mathrm{p}}>300$ & $\begin{array}{l}\text { Particles stray from flowlines by inertia and } \\
\text { collide with fibers }\end{array}$ \\
$E_{\mathrm{G}}$ Gravitational & Very Large & $d_{\mathrm{p}}>500$ & Particles stray from flowlines by force of gravity \\
\hline
\end{tabular}

\section{A.5. Interception Efficiency $E_{R}$}

Interception is a key mechanism for the collection of particles smaller than $0.2 \mu \mathrm{m}$ which generally follow along laminar airflow streamlines through the filter media. As illustrated in Figure A5 particles following streamlines within one particle radius from the fiber surface contact the fiber and are assumed to stick to it.

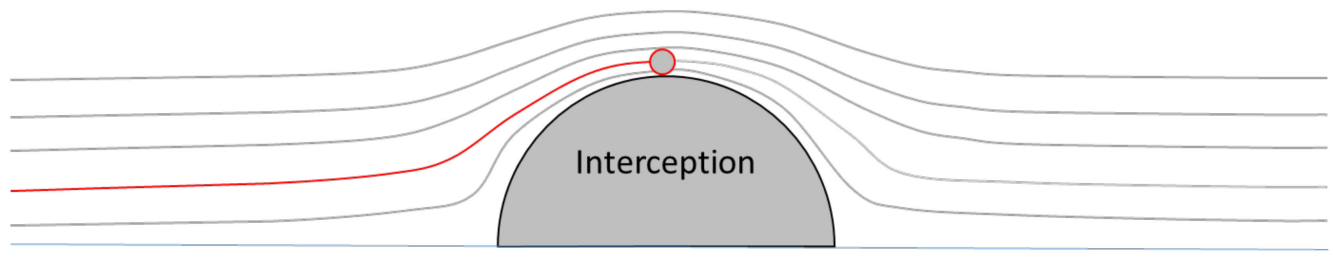

Figure A5. Interception deposition mechanism.

In polar coordinates, the stream flow lines come nearest the fiber surface at $\theta=\frac{\pi}{2}$ where the flow stream lines are parallel to the overall direction of flow. For a given fiber with radius $r_{\mathrm{f}}$ and a given particle size with radius $r_{\mathrm{p}}$, the particle will collide with the fiber if the sum of the particle and fiber radii is greater than the distance from the center of the fiber to the stream flow line at $\theta=\frac{\pi}{2}$. The interception efficiency for a single fiber when considering only the particles geometrically incident to the fiber is therefore shown by Equation (A6).

$$
E_{\mathrm{R}}=\frac{2 y}{2 r_{\mathrm{f}}}=\frac{\psi}{v r_{\mathrm{f}}}
$$

Defining $R$ as the ratio of particle diameter to fiber diameter as shown in Equation (A7), the Interception Efficiency $\left(E_{\mathrm{R}}\right)$ can be written from the stream flow model as shown in Equation (A8) [44].

$$
R=\frac{d_{\mathrm{p}}}{d_{\mathrm{f}}}
$$




$$
E_{\mathrm{R}}=\frac{1+R}{2 \mathrm{Ku}}\left(2 \ln (1+R)-1+\alpha_{\mathrm{f}}+\left(\frac{1}{1+R}\right)^{2}\left(1-\frac{\alpha_{\mathrm{f}}}{2}\right)-\frac{\alpha_{\mathrm{f}}}{2}(1+R)^{2}\right)
$$

However, there have been several efforts to simplify Equation (A8) for ER. One of the most widely used approximations is shown in Equation (A9) [44].

$$
E_{\mathrm{R}}=\frac{\left(1-\alpha_{\mathrm{f}}\right) R^{2}}{(\mathrm{Ku})(1+R)}
$$

Up to this point, this model does not account for slip boundary conditions for very small fibers, which are generally smaller than $2 \mu \mathrm{m}$ in diameter. A useful non-dimensional number commonly used to address slip is the Knudsen number, defined as the ratio of molecular mean free path length of a gas to a characteristic length, such as the diameter or radius of a particle or fiber. The Knudsen number for a fiber is the mean free path of air, $\lambda$, divided by the fiber radius as shown in Equation (A10) (although this form is based upon the fiber diameter). The mean free path of air is approximately $65 \mathrm{~nm}$ at standard conditions.

$$
\mathrm{Kn}=\frac{2 \lambda}{d_{\mathrm{f}}}
$$

The smaller the fiber diameter, the larger the Knudsen number, which is an indicator of the boundary slip condition of an airstream along a fiber surface. Air flow around a fiber falls into one of four categories depending on the Knudsen number as shown in Table A3.

Table A3. Particle capture mechanisms based on particle size.

\begin{tabular}{cccc}
\hline Kn & Category & Fiber Diameter $d_{\mathrm{f}}$ \\
\hline $\mathrm{Kn}<0.01$ & Continuum (Non-Slip) & $d_{\mathrm{f}}>220(\lambda)$ & $d_{\mathrm{f}}>13 \mu \mathrm{m}$ \\
$0.01<\mathrm{Kn}<0.25$ & Slip Flow & $8(\lambda)<d_{\mathrm{f}}<200(\lambda)$ & $520 \mathrm{~nm}<d_{\mathrm{f}}<13 \mu \mathrm{m}$ \\
$0.25<\mathrm{Kn}<10$ & Transient & $0.2(\lambda)<d_{\mathrm{f}}<8(\lambda)$ & $13 \mathrm{~nm}<d_{\mathrm{f}}<520 \mathrm{~nm}$ \\
$10.0<\mathrm{Kn}$ & Free Molecule Range & $d_{\mathrm{f}}<0.2(\lambda)$ & $d_{\mathrm{f}}<13 \mathrm{~nm}$ \\
\hline
\end{tabular}

Most nanofiber filters are in the range of slip flow and transient flow categories, with fiber diameters ranging from $13 \mathrm{~nm}$ to $13 \mu \mathrm{m}$ and Knudsen numbers ranging from 0.01 and 10.00. HEPA filters are mostly in the transient flow category with fiber diameters less than $500 \mathrm{~nm}$. Larger values of $\mathrm{Kn}$ indicate that particles traveling along air flow streamlines are less likely to be influenced and collected by the fibers, resulting in higher penetration and lower pressure drop. It is worth noting that Brownian diffusion (discussed in Appendix A.6) becomes the primary SFE component for particles with large Knudsen numbers, as the aerosol particles have diameter sizes in the range of the mean free path of air molecules.

A simple modification of the Kuwabara hydrodynamic factor to account for slip conditions on the fiber boundary is applied by adding the Knudsen number to the hydrodynamic factor in Equation (A9). The resulting SFE component for interception efficiency is shown in Equation (A11).

$$
E_{\mathrm{R}}=\frac{\left(1-\alpha_{\mathrm{f}}\right) R^{2}}{\left(\mathrm{Ku}+\frac{2 \lambda}{d_{\mathrm{f}}}\right)(1+R)}
$$

\section{A.6. Inertial Impaction Efficiency $E_{I}$}

Inertial impaction plays the primary role in the collection of particles generally larger than $0.3 \mu \mathrm{m}$, and can be neglected for very small particles with low momentum. Momentum and the particle stopping distance are key parameters of inertial impaction. A visual representation of inertial impaction is depicted in Figure A6. 


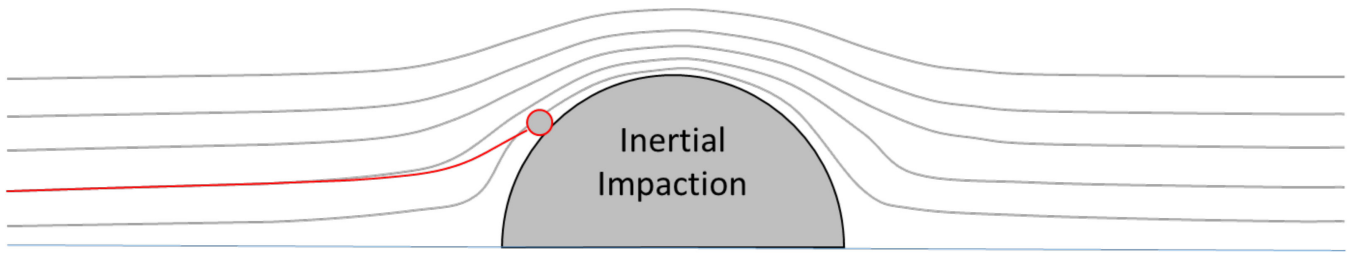

Figure A6. Inertial impaction deposition mechanism.

The Reynolds number earlier described in Appendix A.1 in relation to the fiber and air flow is now considered in relation to the aerosol particle. The Reynolds number is the ratio of inertial force to frictional viscous force as the particle flows through air, which is important for impaction deposition mechanics. The motion of a particle with a low Reynolds number is governed by frictional force, which will cause it to generally follow air flow stream lines. On the other hand, the motion of a particle with high Reynolds number is governed by inertial force which will create a tendency to veer outside of a streamline when the streamline changes in its direction. For high Reynolds number between 1000 and 200,000, Newton's resistance law enables the calculation of the drag force using an empirical coefficient of drag, density, diameter, and velocity of an object. The transition range is for Reynolds number between 1 and 1000. Most aerosols flow with low Reynolds number, less than unity, where viscous forces are greater than inertial forces. Stokes Law becomes important for inertial impaction in the region of Stokes flow, where a significant amount of air filtration takes place. By dismissing inertial forces as negligible and assuming incompressible flow, the Navier-Stokes equations simplify, eliminating higher order terms and yielding solvable linear equations. The force of drag experienced by a particle is shown in Equation (A12).

$$
F_{\mathrm{d}}=3 \pi \eta v_{\mathrm{t}} d_{\mathrm{p}}
$$

The terminal velocity $v_{t}$ can be calculated by equating the drag force of a particle to the force exerted on the same particle, such as the force from gravity. Thus, the particle mobility $B$, illustrated in Equation (A13), is the ratio of the terminal velocity of a particle to the steady force producing that velocity. It is convenient to consider this variable as a relative guide for the particle attaining a steady motion. For example, a large particle mobility could be indicative that a particle will not need to be acted on by a large force to achieve its terminal velocity or that its terminal velocity is high for a given force, whereas a small particle mobility indicates the opposite.

$$
B=\frac{v_{\mathrm{t}}}{F}
$$

Understanding the concept of particle mobility, the relaxation time $\tau$ may be defined as the time required for a particle to adjust to a new velocity for a newly applied force. This is illustrated in Equation (A14), and may be calculated by utilizing the terminal velocity divided by acceleration, or the mass of the particle multiplied by the particle mobility. A particle reaches $63 \%$ of its terminal velocity after its relaxation time, and $95 \%$ of its terminal velocity after three times its relaxation time.

$$
\tau=m B=\frac{\rho_{\mathrm{p}} d_{\mathrm{p}}^{2} C_{\mathrm{c}}}{18 \eta}
$$

Building upon the relaxation time, the concept of a stopping distance may be introduced as the distance a particle will travel with a given initial velocity $v_{0}$ until it stops. The particle's stopping distance $S$ may be calculated if the particle is within the Stokes region using Equation (A15).

$$
S=\tau v_{0}
$$


Finally, curvilinear motion is characterized by the Stokes number, which may be defined as the stopping distance divided by a characteristic dimension. The fiber diameter is typically used in the analysis of fibrous filters as the characteristic dimension, shown in Equation (A16).

$$
\text { Stk }=\frac{S}{d_{\mathrm{c}}}=\frac{\tau U_{0}}{d_{\mathrm{f}}}
$$

The Stokes number is an indication of a particle's ability to change direction and follow along air flow streamlines around a fiber, in the case of Equation (A16). For large Stokes numbers much greater than unity, a particle has adequate inertia to continue in a relatively straight line when the air molecules surrounding it turn, creating a high probability of the particle veering outside the flow line and colliding with a nearby fiber, thus being collected by the inertial impaction mechanism. For low Stokes numbers much less than unity, a particle has insufficient inertia to veer outside flow lines while it moves along with the air flow. In this manner the Stokes number is the measurement of a particle's persistence to stay along a flow streamline in comparison to the size of a fiber. The SFE for Impaction $\left(E_{\mathrm{I}}\right)$ is calculated using $\mathrm{Stk}, \alpha_{\mathrm{f}}$, and $\mathrm{Ku}$ as shown in Equation (A17):

$$
E_{\mathrm{I}}=\frac{(\mathrm{Stk}) J}{2(\mathrm{Ku})^{2}} \text { where } J=\left(29.6-28 \alpha_{\mathrm{f}}^{0.62}\right) R^{2}-27.5 R^{2.8} .
$$

\section{Appendix A.7 Diffusion Efficiency ED}

Diffusion generally plays the role of the primary deposition mechanism for the filtration of particles smaller than $0.3 \mu \mathrm{m}$. Brownian diffusion is the seemingly random motion of particles interacting with the collision energy of the transporting medium. In this, particles tend to veer outside of flow streamlines with seemingly random and erratic movement, thus colliding with and adhering to fibers. This is illustrated in Figure A7 below.

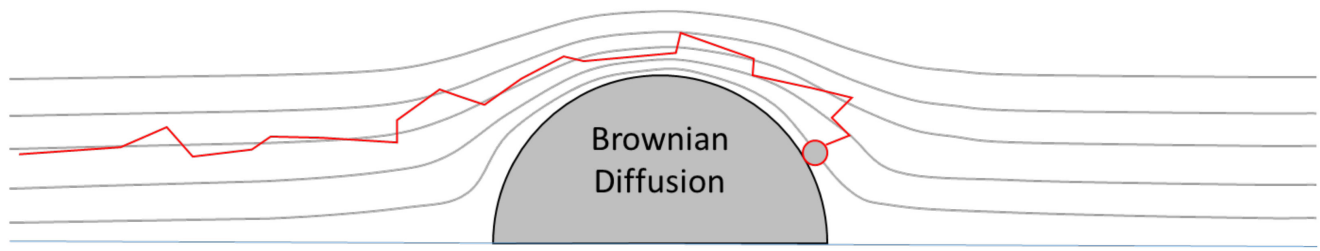

Figure A7. Brownian diffusion deposition mechanism.

However, since Brownian diffusion is known to be the result of many molecular collisions with a particle, it cannot be applied directly to very small particles generally less than $1 \mu \mathrm{m}$ diameter, without being corrected. This is because as described above, Stokes Law cannot account for the dynamics of very small particles capable of slipping between the air molecules due to the particle's very small size. To address this issue, Cunningham developed a slip correction factor for Stokes Law based on the particle diameter and the mean free path of air as shown in Equation (A18).

$$
C_{c}=1+\frac{2.52 \lambda}{d_{p}}
$$

For particles below $100 \mathrm{~nm}$ diameter, an alternative version of the slip correction factor is necessary, and the resulting correction factor is shown in Equation (A19).

$$
C_{\mathrm{c}}=1+\frac{\lambda}{d_{\mathrm{p}}}\left(2.34+1.05 \mathrm{e}^{-0.39 d_{\mathrm{p}} / \lambda}\right)
$$


As the viscosity of the air is a necessary variable in air filtration, it is worthwhile to present a possible equation for its calculation, shown in Equation (A20), using the absolute temperature $T$ and yielding units of $P a \cdot s$.

$$
\eta=\frac{1.458 \mathrm{E}-6 \cdot T^{1.5}}{T+110.4}
$$

The coefficient of diffusion $D$ may now be calculated from the absolute temperature in Kelvin, the Boltzmann constant $k$ of $1.38 \times 10^{-23} \mathrm{~J} / \mathrm{K}$, air viscosity $\eta$, particle diameter $d_{\mathrm{p}}$ and slip correction factor $C_{\mathrm{c}}$ as shown in Equation (A21) with units of $\mathrm{m}^{2} / \mathrm{s}$.

$$
D=\frac{k T C_{\mathrm{c}}}{3 \pi \eta d_{\mathrm{p}}}
$$

Finally, the Peclet number may be defined as a dimensionless ratio of the rate of advection of a quantity to the rate of diffusion of the same quantity. This may be calculated, as shown in Equation (A22), as the product of the velocity $v$ and fiber diameter $d_{\mathrm{f}}$ divided by the particle diffusion coefficient.

$$
\mathrm{Pe}=\frac{(v)\left(d_{\mathrm{f}}\right)}{D}
$$

The single fiber efficiency based on the diffusion of particles may now be calculated from using the Peclet number, as shown below in Equation (A23).

$$
E_{\mathrm{D}}=2 \mathrm{Pe}^{-2 / 3}
$$

\section{Appendix A.8 Diffusion-Interception Efficiency $E_{D R}$}

A common assumption when using the SFE model is that each of the different deposition mechanisms work independently of the others. Although a valid assumption in many cases, it is not always realistic or applicable and thus sometimes an additional efficiency term is added to account for the interaction between the different prevailing deposition mechanisms. Equation (A24) illustrates the efficiency term accounting for the enhanced collection of diffusing particles through the interception mechanism.

$$
E_{\mathrm{DR}}=\frac{1.24 R^{2 / 3}}{(\mathrm{Ku} \cdot \mathrm{Pe})^{1 / 2}}
$$

\section{Appendix A.9 Gravitational Settling Efficiency EG}

Gravity plays a role in air filtration, forcing particles to either enter the airstream or depart from the airstream. The dimensionless constant that governs gravitational settling efficiency is shown in Equation (A25).

$$
G=\frac{V_{\mathrm{TS}}}{U_{0}}=\frac{\rho_{\mathrm{g}} d_{\mathrm{p}}^{2} C_{\mathrm{c} g}}{18 \eta U_{0}}
$$

The efficiency gained or lost by gravitational settling depends on the direction of airflow compared to the direction of gravitational force, given in Equation (A26). If the airflow is in the same direction as gravity, $E_{\mathrm{G}}=G(1+R)$ which has a positive effect on single fiber efficiency. However, if the airflow is in the opposite direction, gravity has a negative effect on single fiber efficiency, as $E_{\mathrm{G}}=-G(1+R)$.

$$
E_{\mathrm{G}}= \pm G(1+R)
$$




\section{Appendix A.10 Total Filter Efficiency}

The single fiber efficiencies based on individual deposition mechanisms described above can be combined into the single fiber efficiency as shown in Equation (A27). As stated previously, this method makes the assumption that each deposition mechanism acts independently of the other deposition mechanisms, although additional terms are sometimes added to address the interaction between them.

$$
E_{\Sigma}=1-\left(1-E_{\mathrm{R}}\right)\left(1-E_{\mathrm{D}}\right)\left(1-E_{\mathrm{DR}}\right)\left(1-E_{\mathrm{I}}\right)\left(1-E_{\mathrm{G}}\right)\left(1-E_{\mathrm{E}}\right)
$$

The total filter penetration $P_{\mathrm{F}}$ may now be calculated for the filter media as a whole using an exponential function with the single fiber efficiency, filter solidity, filter media thickness, and fiber diameter variables as shown in Equation (A28). The total filtration efficiency is then the complement of total filter penetration as shown in Equation (A29) [10]. It is worth specifically stating that these equations for total filter penetration and efficiency are based on a singular particle size, which is factored into the single fiber efficiency $E_{\Sigma}$.

$$
\begin{gathered}
P_{\mathrm{F}}=\exp \left(\frac{-4 \alpha E_{\Sigma} t}{\pi d_{\mathrm{f}}}\right) . \\
E_{\mathrm{F}}=1-P_{\mathrm{F}}=1-\exp \left(\frac{-4 \alpha E_{\Sigma} t}{\pi d_{\mathrm{f}}}\right)
\end{gathered}
$$

A representation of component single fiber efficiencies and total filter efficiency is over a range of particle diameters is shown in Figure A8.

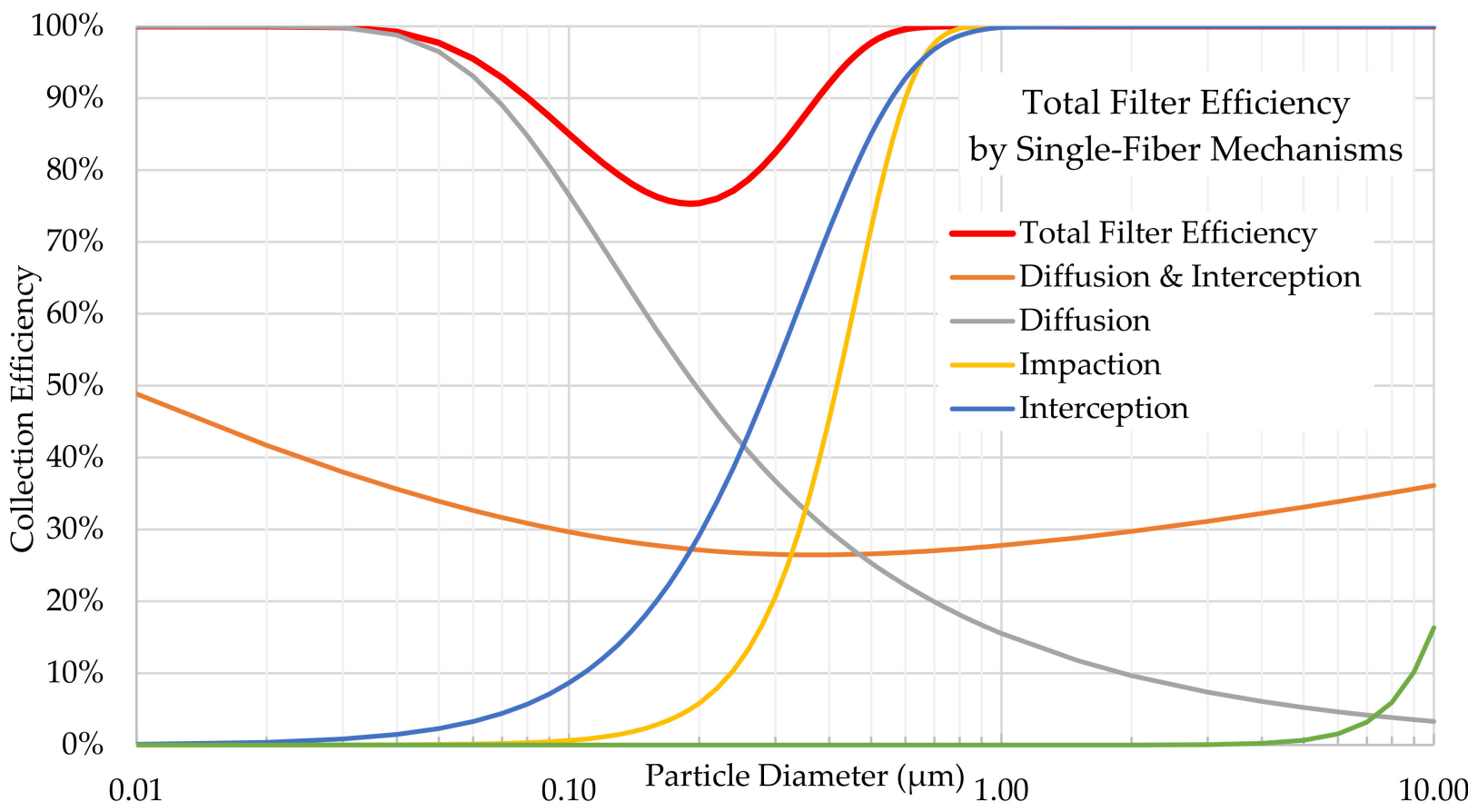

Figure A8. Total filter efficiency by single fiber mechanisms.

The single fiber efficiency model shown here is dependent on known particle sizes to calculate the MPPS and the total filter efficiency. However, Lee and Liu developed a set of equations in 1980 to predict minimum single fiber efficiency and the most-penetrating particle size based solely on the interception and diffusion deposition mechanisms, shown in Equations A30 and A31 [43]. The minimum single fiber efficiency from Equation (A30) 
may be substituted into Equation (A29) above to calculate the total filter efficiency if the analysis is conducted with regard to the MPPS.

$$
\begin{gathered}
\hat{E_{\Sigma}}=1.44\left[\left(\frac{1-\alpha}{\mathrm{Ku}}\right)^{5}\left(\frac{\sqrt{\lambda} k T}{\eta}\right)^{4}\left(\frac{1}{U_{0}^{4} d_{\mathrm{f}}^{10}}\right)\right]^{1 / 9} \\
\hat{d_{\mathrm{p}}}=0.885\left[\left(\frac{\mathrm{Ku}}{1-\alpha}\right)\left(\frac{\sqrt{\lambda} k T}{\eta}\right)\left(\frac{d_{\mathrm{f}}^{2}}{U_{0}}\right)\right]^{2 / 9}
\end{gathered}
$$

Appendix A.11 Air Flow Resistance

The flow resistance through a filter media, measured by pressure drop across the media, is equally as important as the filtration efficiency when calculating the filter's overall FOM rating. The goal of filter design is to achieve low flow resistance with high filtration efficiency. Pressure drop across a filter is directly proportional to the air viscosity, air face velocity, filter thickness, and the filter's solid volume fraction. Furthermore, flow resistance is inversely proportional to the square of the fiber diameter as shown in Equation (A32). Many authors have worked on creating realistic models through analytical, numerical, and empirical means. Conveniently, many of these may be incorporated into Equation (A32) through a dimensionless function of the solidity, $f\left(\alpha_{\mathrm{f}}\right)$. The work of a few authors is included in Table A4.

$$
\Delta P=\frac{\eta t U_{0}}{d_{\mathrm{f}}^{2}} f\left(\alpha_{\mathrm{f}}\right)
$$

Table A4. Flow resistance coefficients based on solidity for various authors.

\begin{tabular}{cc}
\hline Author & $f\left(\boldsymbol{\alpha}_{\mathrm{f}}\right)$ \\
\hline Happel [23] & $-\frac{32 \alpha_{\mathrm{f}}}{\left[\ln \left(\alpha_{\mathrm{f}}\right)+\frac{\left(1-\alpha_{\mathrm{f}}\right)^{2}}{\left(1+\alpha_{\mathrm{f}}\right)^{2}}\right]}$ \\
Kuwabara [23] & $-\frac{4 \alpha_{\mathrm{f}}}{\left[2 \ln \left(\alpha_{\mathrm{f}}\right)+3-4 \alpha_{\mathrm{f}}+\alpha_{\mathrm{f}}{ }^{2}\right]}$ \\
Davies [10] & $64 \alpha_{\mathrm{f}}^{1.5}\left(1+56 \alpha_{\mathrm{f}}^{3}\right)$ \\
Henry and Ariman [41] & $2.446 \alpha_{\mathrm{f}}+38.16 \alpha_{\mathrm{f}}{ }^{2}+138.9 \alpha_{\mathrm{f}}^{3}$ \\
Rao and Faghri [39] & $2.653 \alpha_{\mathrm{f}}+39.34 \alpha_{\mathrm{f}}{ }^{2}+144.5 \alpha_{\mathrm{f}}{ }^{3}$ \\
\hline
\end{tabular}

\section{Appendix A.12 Effective Fiber Diameter}

It is worth noting here that the equations for calculating the pressure drop may be manipulated in order to estimate the effective fiber diameter of the filtration media, if the other variables in the equation are known. The effective fiber diameter may then be calculated as:

$$
d_{\mathrm{fe}}=\sqrt{\frac{\eta t U_{0} f\left(\alpha_{\mathrm{f}}\right)}{\Delta P}}
$$

\section{Appendix A.13 Filter Figure of Merit (FOM)}

Ultimately, the objective of air filtration is to maximize the collection efficiency for a filter while minimizing the flow resistance. The quality of a filter may be thought of as a comparison of the particle collection efficiency to flow resistance. The filter Figure of Merit (FOM), also known as the Quality Factor (QF), is shown in Equation (A34) with units of inverse Pascal.

$$
\mathrm{FOM}=\frac{-\ln \left(P_{\mathrm{F}}\right)}{\Delta P}=\frac{-\ln \left(1-E_{\mathrm{F}}\right)}{\Delta P}
$$


Table A5. List of terms, symbols, and units in the analytical model.

\begin{tabular}{|c|c|c|}
\hline Term & Description & $\begin{array}{c}\text { Unit of } \\
\text { Measurement }\end{array}$ \\
\hline$A$ & Filter media cross sectional area & $\mathrm{m}^{2}$ \\
\hline$B$ & Mobility & $\mathrm{s} / \mathrm{kg}$ \\
\hline$b$ & Distance to boundary (Kuwabara model) & $\mathrm{m}$ \\
\hline$C_{c}$ & Cunningham slip correction factor & dimensionless \\
\hline$c_{\text {in }}$ & Count of particles approaching fiber & dimensionless \\
\hline$c_{\text {out }}$ & Count of particles escaping by fiber & dimensionless \\
\hline$D$ & Diffusion coefficient & $\mathrm{m}^{2} / \mathrm{s}$ \\
\hline$d_{\mathrm{c}}$ & Characteristic dimension & $\mathrm{m}$ \\
\hline$d_{\mathrm{f}}$ & Fiber diameter & $\mathrm{m}$ \\
\hline$d_{\mathrm{fe}}$ & Effective fiber diameter & $\mathrm{m}$ \\
\hline$d_{\mathrm{p}}$ & Aerosol particle diameter & $\mathrm{m}$ \\
\hline$\hat{d_{\mathrm{p}}}$ & Most penetrating particle size & $\mathrm{m}$ \\
\hline$E_{\Sigma}$ & Combined single fiber efficiency of components & dimensionless \\
\hline$\hat{E_{\Sigma}}$ & Minimum SFE (Lee and Liu) & dimensionless \\
\hline$E_{\mathrm{D}}$ & SFE diffusion component & dimensionless \\
\hline$E_{\mathrm{DR}}$ & SFE for interception of diffusing particles & dimensionless \\
\hline$E_{\mathrm{E}}$ & SFE electrostatic component & dimensionless \\
\hline$E_{\mathrm{F}}$ & Total Filter Efficiency & dimensionless \\
\hline$E_{\mathrm{G}}$ & SFE gravity component & dimensionless \\
\hline$E_{\mathrm{I}}$ & SFE inertial impaction component & dimensionless \\
\hline$E_{\mathrm{R}}$ & SFE interception component & dimensionless \\
\hline$F_{\mathrm{d}}$ & Force of drag on a particle & $\mathrm{N}$ \\
\hline FOM & Figure of Merit, also Quality Factor & $\mathrm{Pa}^{-1}$ \\
\hline G & Gravitational coefficient for SFE component & dimensionless \\
\hline$g$ & Gravitational constant $(9.81)$ & $\mathrm{m} / \mathrm{s}^{2}$ \\
\hline Kn & Knudsen number & dimensionless \\
\hline $\mathrm{Ku}$ & Kuwabara hydrodynamic factor & dimensionless \\
\hline$k$ & Boltzmann constant, $1.38 \times 10^{-23} \mathrm{~J} / \mathrm{K}$ & $\mathrm{J} / \mathrm{K}$ \\
\hline$m$ & Mass of the particle & $\mathrm{kg}$ \\
\hline$P_{\Sigma}$ & Single fiber penetration & dimensionless \\
\hline$P_{\mathrm{F}}$ & Total filter penetration & dimensionless \\
\hline $\mathrm{Pe}$ & Peclet number & dimensionless \\
\hline$Q$ & Volumetric flow rate of air & $\mathrm{m}^{3} / \mathrm{s}$ \\
\hline$\widetilde{R}$ & Ratio of particle-to-fiber diameter & dimensionless \\
\hline $\operatorname{Re}$ & Reynolds number & dimensionless \\
\hline$r_{\mathrm{f}}$ & Fiber radius & $\mathrm{m}$ \\
\hline$r_{\mathrm{p}}$ & Particle radius & $\mathrm{m}$ \\
\hline S & Particle stopping distance & $\mathrm{m}$ \\
\hline Stk & Stokes number & dimensionless \\
\hline$T$ & Absolute Temperature & $\mathrm{K}$ \\
\hline$t$ & Thickness of the air filter media & $\mathrm{m}$ \\
\hline$U_{0}$ & Air velocity & $\mathrm{m} / \mathrm{s}$ \\
\hline$u_{\mathrm{r}}$ & Radial velocity (Kuwabara model) & $\mathrm{m} / \mathrm{s}$ \\
\hline$u_{\theta}$ & Tangential velocity (Kuwabara model) & $\mathrm{m} / \mathrm{s}$ \\
\hline$\alpha_{\mathrm{f}}$ & Solidity, solid volume fraction, packing density & dimensionless \\
\hline$\Delta P$ & Pressure differential, flow resistance & $\mathrm{Pa}$ \\
\hline$\eta$ & Air viscosity & $\mathrm{Pa} \cdot \mathrm{s}$ \\
\hline$\lambda$ & Mean free path of air, approximately $65 \mathrm{~nm}$ & $\mathrm{~m}$ \\
\hline$v$ & Mean air velocity inside cell (Kuwabara model) & $\mathrm{m} / \mathrm{s}$ \\
\hline$v_{0}$ & Initial velocity of a particle (mobility) & $\mathrm{m} / \mathrm{s}$ \\
\hline$v_{\mathrm{t}}$ & Terminal velocity of a particle (mobility) & $\mathrm{m} / \mathrm{s}$ \\
\hline$\rho_{\mathrm{g}}$ & Air density & $\mathrm{kg} / \mathrm{m}^{3}$ \\
\hline$\rho_{\mathrm{p}}$ & Density of the particle & $\mathrm{kg} / \mathrm{m}^{3}$ \\
\hline$\tau$ & Relaxation time & $\mathrm{s}$ \\
\hline$\psi$ & Stream function (Kuwabara model) & dimensionless \\
\hline$\omega$ & Vorticity (Kuwabara model) & rotations $/ \mathrm{s}$ \\
\hline$\nabla^{2}$ & Vector Laplacian (Kuwabara model) & dimensionless \\
\hline
\end{tabular}


Table A6. Example calculations.

\begin{tabular}{|c|c|c|c|}
\hline Term & Description & Value & SI Units \\
\hline$A$ & Filter cross sectional area & $1.00 \mathrm{~m}^{2}$ & $1.00 \mathrm{~m}^{2}$ \\
\hline$Q$ & Volumetric flow rate of air & $6.00 \mathrm{~m}^{3} / \mathrm{min}$ & $1.00 \times 10^{-1} \mathrm{~m}^{3} / \mathrm{s}$ \\
\hline$U_{0}$ & Air velocity & $10.0 \mathrm{~cm} / \mathrm{s}$ & $1.00 \times 10^{-1} \mathrm{~m} / \mathrm{s}$ \\
\hline$d_{\mathrm{f}}$ & Fiber diameter & $2.0 \mu \mathrm{m}$ & $2.00 \times 10^{-6} \mathrm{~m}$ \\
\hline$r_{\mathrm{f}}$ & Fiber radius & $1.0 \mu \mathrm{m}$ & $1.00 \times 10^{-6} \mathrm{~m}$ \\
\hline$d_{\mathrm{p}}$ & Particle diameter & $300 \mathrm{~nm}$ & $3.00 \times 10^{-7} \mathrm{~m}$ \\
\hline$r_{\mathrm{p}}$ & Particle radius & $150 \mathrm{~nm}$ & $1.50 \times 10^{-7} \mathrm{~m}$ \\
\hline$R$ & Particle-to-fiber ratio & $15 \%$ & \\
\hline$\rho_{\mathrm{p}}$ & Particle density & $1.0 \mathrm{~g} / \mathrm{cm}^{3}$ & $1.00 \times 10^{3} \mathrm{~kg} / \mathrm{m}^{3}$ \\
\hline$m$ & Particle mass & $14.14 \mathrm{ag}$ & $1.414 \times 10^{-17} \mathrm{~kg}$ \\
\hline$\lambda$ & Mean free path of air & $65.3 \mathrm{~nm}$ & $6.53 \times 10^{-8} \mathrm{~m}$ \\
\hline$k$ & Boltzmann constant & $1.38 \times 10^{-23} \mathrm{~J} / \mathrm{K}$ & $1.38 \times 10^{-23} \mathrm{~J} / \mathrm{K}$ \\
\hline$T$ & Absolute Temperature & $293 \mathrm{~K}$ & $2.93 \times 10^{2} \mathrm{~K}$ \\
\hline$\rho_{\mathrm{g}}$ & Air density & $1.2 \mathrm{~kg} / \mathrm{m}^{3}$ & $1.2 \mathrm{~kg} / \mathrm{m}^{3}$ \\
\hline$g$ & Gravitational constant & $9.81 \mathrm{~m} / \mathrm{s}^{2}$ & $9.81 \mathrm{~m} / \mathrm{s}^{2}$ \\
\hline & Efficiency Calculations & & \\
\hline$\eta$ & Air viscosity & $1.813 \times 10^{-5} \mathrm{~Pa} \cdot \mathrm{s}$ & $1.813 \times 10^{-5} \mathrm{~Pa} \cdot \mathrm{s}$ \\
\hline $\operatorname{Re}$ & Reynolds number & 0.01324 & \\
\hline $\mathrm{Ku}$ & Hydrodynamic factor & 0.797 & \\
\hline \multirow[t]{2}{*}{$\mathrm{Kn}$} & Knudsen number & 0.0653 & \\
\hline & Ratio $\lambda / d_{\mathrm{p}}$ & 0.218 & \\
\hline$E_{\mathrm{R}}$ & SFE interception & 0.0233 & \\
\hline$C_{\mathrm{c}}$ & Slip correction factor & 1.547 & \\
\hline$D$ & Diffusion coefficient & $1.221 \times 10^{-10} \mathrm{~m}^{2} / \mathrm{s}$ & $1.221 \times 10^{-10} \mathrm{~m}^{2} / \mathrm{s}$ \\
\hline $\mathrm{Pe}$ & Peclet number & 1638.2 & \\
\hline$E_{\mathrm{D}}$ & SFE diffusion & 0.01439 & \\
\hline$E_{\mathrm{DR}}$ & Diffusion interception & $9.69 \times 10^{-3}$ & \\
\hline$F_{\mathrm{d}}$ & Force of drag on particle & $5.13 \mathrm{pN}$ & $5.13 \times 10^{-12} \mathrm{~N}$ \\
\hline$B$ & Mobility & $3.02 \times 10^{10} \mathrm{~s} / \mathrm{kg}$ & $3.02 \times 10^{10} \mathrm{~s} / \mathrm{kg}$ \\
\hline$\tau$ & Relaxation time & $427 \mathrm{~ns}$ & $4.27 \times 10^{-7} \mathrm{~s}$ \\
\hline$S$ & Particle stopping distance & $42.7 \mathrm{~nm}$ & $4.27 \times 10^{-8} \mathrm{~m}$ \\
\hline \multirow[t]{2}{*}{ Stk } & Stokes number & 0.02134 & \\
\hline & $J$ factor & 0.432 & \\
\hline$E_{\mathrm{I}}$ & SFE inertial impaction & 0.00725 & \\
\hline$v_{\mathrm{t}}$ & Terminal velocity & $4.19 \mu \mathrm{m} / \mathrm{s}$ & $4.19 \times 10^{-6} \mathrm{~m} / \mathrm{s}$ \\
\hline G & Gravitational coefficient & $4.19 \times 10^{-5}$ & \\
\hline$E_{\mathrm{G}}$ & SFE gravity component & $2.02 \times 10^{-9}$ & \\
\hline$E_{\Sigma}$ & Single fiber efficiency & $5.46 \%$ & \\
\hline$P_{\Sigma}$ & Single fiber penetration & $94.54 \%$ & \\
\hline$P_{\mathrm{F}}$ & Total filter penetration & $17.6 \%$ & \\
\hline \multirow[t]{3}{*}{$E_{\mathrm{F}}$} & Total filter efficiency & $82.4 \%$ & \\
\hline & Pressure Drop Calculation & & \\
\hline & Davies model $f\left(\alpha_{\mathrm{f}}\right)$ & 0.7206 & \\
\hline \multirow[t]{2}{*}{$\Delta P$} & Pressure differential & $326.5 \mathrm{~Pa}$ & $3.265 \times 10^{2} \mathrm{~Pa}$ \\
\hline & Figure of Merit Calculation & & \\
\hline FOM & Figure of Merit & $0.00306 \mathrm{~Pa}^{-1}$ & $3.06 \times 10^{-3} \mathrm{~Pa}^{-1}$ \\
\hline
\end{tabular}

\section{References}

1. Berry, G.; Parsons, A.; Morgan, M.; Rickert, J.; Cho, H. A review of methods to reduce the probability of the airborne spread of COVID-19 in ventilation systems and enclosed spaces. Environ. Res. 2022, 203, 111765. [CrossRef]

2. U.S. Department of Energy. Nuclear Air Cleaning Handbook, 4th ed. 2003. Available online: https://www.standards.doe.gov/ standards-documents/1100/1169-bhdbk-2003-ch2/@@images/file (accessed on 29 October 2021).

3. Bergman, W.; Taylor, R.D.; Miller, H.H.; Bierman, A.H.; Hebard, H.D.; Daroza, R.A.; Lum, B.Y. Enhanced Filtration Program at LLL-A Progress Report. In Proceedings of the 15th DOE/NRC Nuclear Air Cleaning and Treatment Conference, Boston, MA, USA, 1 August 1978.

4. Haslam, J.J.; Mitchell, M.A. Ceramic Filter with Nanofibers. US 2013/0048579 A1, 28 February 2013. 
5. Bogle, B.; Kelly, J.; Haslam, J. Transient Heating and Thermomechanical Stress Modeling of Ceramic HEPA Filters; Lawrence Livermore National Lab. (LLNL): Livermore, CA, USA, 2017. [CrossRef]

6. Kelly, J.P.; Haslam, J.J.; Mitchell, M.A.; Makeswaran, N.; Maguire, J.; Finkenauer, L. NSRD-12, Novel Mini-Tubular HEPA Media for Nuclear Facility Ventilation Systems; Lawrence Livermore National Lab. (LLNL): Livermore, CA, USA, 2018. [CrossRef]

7. Mitchell, M.; Bergman, W.; Haslam, J. Ceramic HEPA Filter Program. In Proceedings of the International Society for Nuclear Air Treatment Technologies 32nd Nuclear Air Cleaning Conference, Denver, CO, USA, 7-11 May 2012.

8. Hwang, S.; Roh, J.; Park, W.M. Comparison of the relative performance efficiencies of melt-blown and glass fiber filter media for managing fine particles. Aerosol Sci. Technol. 2018, 52, 451-458. [CrossRef]

9. Davies, C.N. Air Filtration; Academic Press: Cambridge, MA, USA, 1973.

10. Hinds, W.C. Aerosol Technology: Properties, Behavior, and Measurement of Airborne Particles, 2nd ed.; Wiley: New York, NY, USA, 1999.

11. Brown, R.C. Air Filtration: An Integrated Approach to the Theory and Applications of Fibrous Filters; Pergamon Press: Oxford, UK; New York, NY, USA, 1993.

12. Saleh, A.; Tafreshi, H.V.; Pourdeyhimi, B. An analytical approach to predict pressure drop and collection efficiency of dust-load pleated filters. Sep. Purif. Technol. 2016, 161, 80-87. [CrossRef]

13. Saleh, A.; Hosseini, S.; Tafreshi, H.V.; Pourdeyhimi, B. 3-D microscale simulation of dust-loading in thin flat-sheet filters: A comparison with 1-D macroscale simulations. Chem. Eng. Sci. 2013, 99, 284-291. [CrossRef]

14. Yousefi, S.H.; Tang, C.; Tafreshi, H.V.; Pourdeyhimi, B. Empirical model to simulate morphology of electrospun polycaprolactone mats. J. Appl. Polym. Sci. 2019, 136, 48242. [CrossRef]

15. Moghadam, A.; Yousefi, S.H.; Tafreshi, H.V.; Pourdeyhimi, B. Characterizing nonwoven materials via realistic microstructural modeling. Sep. Purif. Technol. 2019, 211, 602-609. [CrossRef]

16. Payatakes, A.; Gradon, L. Dendritic deposition of aerosol particles in fibrous media by inertial impaction and interception. Chem. Eng. Sci. 1980, 35, 1083-1096. [CrossRef]

17. Kuwabara, S. The Forces experienced by Randomly Distributed Parallel Circular Cylinders or Spheres in a Viscous Flow at Small Reynolds Numbers. J. Phys. Soc. JPN. 1959, 14, 527-532. [CrossRef]

18. Lee, K.W.; Liu, B.Y.H. Theoretical Study of Aerosol Filtration by Fibrous Filters. Aerosol Sci. Technol. 1982, 1, 147-161. [CrossRef]

19. Moelter, W.; Fissan, H. Structure of a High Efficiency Glass Fiber Filter Medium. Aerosol Sci. Technol. 1997, 27, 447-461. [CrossRef]

20. Happel, J. Viscous flow relative to arrays of cylinders. AIChE J. 1959, 5, 174-177. [CrossRef]

21. Spielman, L.; Goren, S.L. Model for predicting pressure drop and filtration efficiency in fibrous media. Environ. Sci. Technol. 1968, 2, 279-287. [CrossRef]

22. Faessel, M.; Delisée, C.; Bos, F.; Castéra, P. 3D Modelling of random cellulosic fibrous networks based on X-ray tomography and image analysis. Compos. Sci. Technol. 2005, 65, 1931-1940. [CrossRef]

23. Wang, Q.; Maze, B.; Tafreshi, H.V.; Pourdeyhimi, B. A case study of simulating submicron aerosol filtration via lightweight spun-bonded filter media. Chem. Eng. Sci. 2006, 61, 4871-4883. [CrossRef]

24. Wang, Q.; Maze, B.; Tafreshi, H.V.; Pourdeyhimi, B. Simulating through-plane permeability of fibrous materials with different fiber lengths. Model. Simul. Mater. Sci. Eng. 2007, 15, 855-868. [CrossRef]

25. Maze, B.; Tafreshi, H.V.; Pourdeyhimi, B. Geometrical modeling of fibrous materials under compression. J. Appl. Phys. 2007, 102, 073533. [CrossRef]

26. Maze, B.; Tafreshi, H.V.; Wang, Q.; Pourdeyhimi, B. A simulation of unsteady-state filtration via nanofiber media at reduced operating pressures. J. Aerosol Sci. 2007, 38, 550-571. [CrossRef]

27. Hosseini, S.; Tafreshi, H.V. 3-D simulation of particle filtration in electrospun nanofibrous filters. Powder Technol. 2010, 201, 153-160. [CrossRef]

28. Fotovati, S.; Tafreshi, H.V.; Pourdeyhimi, B. Influence of fiber orientation distribution on performance of aerosol filtration media. Chem. Eng. Sci. 2010, 65, 5285-5293. [CrossRef]

29. Karakoç, A.; Hiltunen, E.; Paltakari, J. Geometrical and spatial effects on fiber network connectivity. Compos. Struct. 2017, 168, 335-344. [CrossRef]

30. Yousefi, S.H.; Tafreshi, H.V. Modeling electrospun fibrous structures with embedded spacer particles: Application to aerosol filtration. Sep. Purif. Technol. 2020, 235, 116184. [CrossRef]

31. Yousefi, S.H.; Venkateshan, D.G.; Tang, C.; Tafreshi, H.V.; Pourdeyhimi, B. Effects of electrospinning conditions on microstructural properties of polystyrene fibrous materials. J. Appl. Phys. 2018, 124, 235307. [CrossRef]

32. Tafreshi, H.V.; Rahman, M.A.; Jaganathan, S.; Wang, Q.; Pourdeyhimi, B. Analytical expressions for predicting permeability of bimodal fibrous porous media. Chem. Eng. Sci. 2009, 64, 1154-1159. [CrossRef]

33. Gervais, P.-C.; Bardin-Monnier, N.; Thomas, D. Permeability modeling of fibrous media with bimodal fiber size distribution. Chem. Eng. Sci. 2012, 73, 239-248. [CrossRef]

34. Mead-Hunter, R.; King, A.J.; Kasper, G.; Mullins, B.J. Computational fluid dynamics (CFD) simulation of liquid aerosol coalescing filters. J. Aerosol Sci. 2013, 61, 36-49. [CrossRef]

35. Grothaus, M.; Klar, A.; Maringer, J.; Stilgenbauer, P.; Wegener, R. Application of a three-dimensional fiber lay-down model to non-woven production processes. J. Math. Ind. 2014, 4, 4. [CrossRef]

36. Abishek, S.; King, A.; Mead-Hunter, R.; Golkarfard, V.; Heikamp, W.; Mullins, B. Generation and validation of virtual nonwoven, foam and knitted filter (separator/coalescer) geometries for CFD simulations. Sep. Purif. Technol. 2017, 188, 493-507. [CrossRef] 
37. American Society of Mechanical Engineers. Code on Nuclear Air and Gas Treatment ASME AG-1-2019; ASME: New York, NY, USA, 2020.

38. Beckman, I.; Lozano, C.; Freeman, E.; Riveros, G. Fiber Selection for Reinforced Additive Manufacturing. Polymers 2021, 13, 2231. [CrossRef]

39. Rao, N.; Faghri, M. Computer Modeling of Aerosol Filtration by Fibrous Filters. Aerosol Sci. Technol. 1988, 8, 133-156. [CrossRef]

40. Kouropoulos, G. The Effect of the Reynolds number of air flow to the particle collection efficiency of a fibrous filter medium with cylindrical section. J. Urban Environ. Eng. 2014, 8, 3-10. [CrossRef]

41. Henry, F.S.; Ariman, T. An Evaluation of the Kuwabara Model. Part. Sci. Technol. 1983, 1, 1-20. [CrossRef]

42. Lee, K.W.; Liu, B.Y.H. Experimental Study of Aerosol Filtration by Fibrous Filters. Aerosol Sci. Technol. 1981, 1, 35-46. [CrossRef]

43. Lee, K.W.; Liu, B.Y.H. On the Minimum Efficiency and the Most Penetrating Particle Size for Fibrous Filters. J. Air Pollut. Control Assoc. 1980, 30, 377-381. [CrossRef]

44. Wang, Q. Investigation of Aerosol Filtratoin via Fibrous Filters. NC State University Libraries, 6 November 2008. Available online: https: / / repository.lib.ncsu.edu/handle/1840.16/5338 (accessed on 29 October 2021). 